\title{
A refined volume-of-fluid algorithm for capturing sharp fluid interfaces on arbitrary meshes
}

\author{
Di Zhang ${ }^{1, *}$, Chunbo Jiang ${ }^{1, *}$, Dongfang Liang ${ }^{2}$, Zhengbing Chen ${ }^{1}$, \\ Yan Yang ${ }^{1}$ and Ying Shi ${ }^{1}$ \\ 1 State Key Laboratory of Hydroscience and Engineering, Tsinghua University, \\ Beijing 100084, China \\ 2 Department of Engineering, University of Cambridge, Trumpington Street, \\ Cambridge CB2 1PZ, UK
}

\begin{abstract}
SUMMARY
This paper presents a new volume-of-fluid scheme (M-CICSAM), capable of capturing abrupt interfaces on meshes of arbitrary topology, which is a modification to the Compressive Interface Capturing Scheme for Arbitrary Meshes (CICSAM) proposed in the recent literature. Without resort to any explicit interface reconstruction, M-CICSAM is able to precisely model the complex free surface deformation, such as interface rupture and coalescence. By theoretical analysis, it is shown that the modified CICSAM overcomes three inherent drawbacks of the original CICSAM, concerning the basic differencing schemes, the switching strategy between the compressive downwind and diffusive high-resolution schemes, and the far-upwind reconstruction technique on arbitrary unstructured meshes. To evaluate the performance of the newly proposed scheme, several classic interface capturing methods developed in the past decades are compared with M-CICSAM. The numerical results clearly demonstrate that M-CICSAM produces more accurate predictions on arbitrary meshes, especially at high Courant numbers, by reducing the numerical diffusion and preserving the interface shape.
\end{abstract}

Keywords: two-phase flows; interface capturing; unstructured meshes; volume of fluid (VOF); normalized variable diagram (NVD); normalized variable and space formulation (NVSF)

\footnotetext{
* Corresponding authors: State Key Laboratory of Hydroscience and Engineering, Tsinghua University, The Hydraulic Building, Beijing 100084, China. Phone: +86 01062781820.

E-mail address: zhangdi10@mails.tsinghua.edu.cn (D. Zhang), jcb@ mail.tsinghua.edu.cn (C. Jiang).
} 


\section{INTRODUCTION}

Numerical simulation of immiscible multi-fluid flows has been an active area of research over the past decades, due to the common presence of such flows in nature and their importance to many practical applications [1-3]. An effective numerical scheme should be able to handle a number of distinct flow features properly, such as high density ratios, large pressure differences at fluid-fluid interfaces, and the evolution of the free surface. Various numerical approaches for computing immiscible multi-fluid flows have been proposed in the literature, which can be grouped into three broad categories: interface/surface fitting methods, interface/ surface tracking methods and interface/surface capturing methods [4-6].

Interface/surface fitting methods [7-11] are associated with boundary-fitted moving grids, where the grid points are attached to the fluid particles and move with them in a Lagrangian manner. Generally, these methods solve for the flow only within a single fluid region and treat the free surface as a free-floating boundary. The advantage of these methods is that they allow a precise representation of the interfacial jump conditions and thus maintain a sharp interface, whose exact position is known throughout the calculation. However, large interface deformations will result in highly distorted meshes. Therefore, special procedures need to be designed in order to prevent both grid singularities and extremely skewed grid point distributions, which impose restrictions on their applications. Both interface tracking and interface capturing methods adopt fixed Eulerian meshes and are particularly well-suited for dealing with large interface deformations [12-13].

In interface/surface tracking methods, such as mark and cell (MAC) schemes and geometric-type VOF schemes, the interface is explicitly approximated either by massless marker particles or by an indicator function. In the MAC methods, the freesurface location is determined by introducing massless marker particles that move with the instantaneous velocity field. In general, such methods are non-conservative and computationally expensive [14-16]. In the geometric-type VOF methods, the interface is explicitly reconstructed by volume fraction values, which is also used in designing the scalar advection scheme. Different researchers have proposed various algorithms of this kind, such as the Simple Line Interface Calculation (SLIC) method and the Piecewise Linear Interface Calculation (PLIC) method [17-21]. These methods are capable of maintaining a very sharp interface while at the same time ensuring mass conservation. However, the processes of reconstructing and tracking 
the interface at each time step remain complicated and challenging, especially when extending to unstructured meshes and to three dimensions.

Interface/surface capturing methods, such as artificial compressibility schemes, level set schemes and algebraic-type VOF schemes, are characterized by algebraically solving the scalar transport equation without explicitly reconstructing the interface, which overcome the main drawbacks of interface tracking methods. In artificial compressibility schemes $[4,6,22]$, the free surface location is automatically captured as a contact discontinuity in the density field by enforcing conservation laws, thus eliminating the need for reconstructing and tracking the interface. In level set schemes [23-24], the interface is defined as the zero-contour of a distance function, which is advected with the local flow field. Although being conceptually simple and relatively easy to implement, level set techniques suffer from the errors in the mass conservation principle.

Algebraic-type VOF methods include high-resolution differencing schemes, fluxlimited methods, inter-gamma schemes, analytical-function fitted methods, blended high-resolution differencing methods. High-resolution differencing schemes [2, 25-27] are inclined to introduce excessive numerical diffusion and dispersion. In intergamma schemes [28-29], an extra artificial compressive term is added into the VOF advection equation for the purpose of compressing the interface, instead of just employing a compressive differencing scheme. Flux-limited methods [30-32] consist of a basic high-resolution advection scheme and a multi-dimensional flux limiter, which are conservative, monotonic and shape-preserving for both continuous and discontinuous density fields. In analytical-function fitted methods [33-34], different smooth basic functions, such as the hyperbolic tangent function and the cubic polynomial function, are adopted to represent a discontinuity at the grid scale in the flux computation of the volume fraction.

A compressive downwind scheme can maintain the sharpness of the interface, but it tends to distort the interface when the flow is not aligned with the computational mesh. On the contrary, a more diffusive high-resolution scheme often turns out to be too diffusive. In order to remedy the aforementioned two problems, blended high-resolution differencing methods make use of the NVD/NVSF concept and switch continuously between a compressive downwind and a diffusive highresolution scheme, according to the angle between the interface direction and the grid orientation. Various blended high-resolution methods have been proposed in the 
literature, including CICSAM [1], HiRAC [3], STACS [5], SURFER [35], HRIC [36], FBICS [37] and THOR [38]. These methods are computationally efficient, strictly conserve mass, and can be easily extended to unstructured meshes and three dimensions, so they are capable of accurately capturing the free surface and modelling merging and fragmentation in multiphase flows.

In this article, a new blended high-resolution differencing method (M-CICSAM) is presented, whose accuracy is compared with four existing schemes of the same kind. The results obtained in all the test cases clearly show the advantage of M-CICSAM.

\section{THE GOVERNING EQUATIONS}

The computational grids across the interface are occupied by different immiscible fluids, which are treated as a single effective fluid with continuous physical properties. The density $\rho$ and dynamic viscosity $\mu$, across the interface are evaluated using the following relations:

$$
\rho=\sum_{i=1}^{n} \alpha_{i} \rho_{i} ; \quad \mu=\sum_{i=1}^{n} \alpha_{i} \mu_{i} ; \quad \sum_{i=1}^{n} \alpha_{i}=1
$$

where the subscript $i$ denotes the $i^{\text {th }}$ fluid, and $\alpha$ is the volume fraction defined as the volume percentage of the $i^{\text {th }}$ fluid available in a cell. The effective fluid is presumed to obey the same set of governing equations as a single fluid:

$$
\begin{gathered}
\frac{\partial \rho}{\partial t}+\frac{\partial \rho u_{i}}{\partial x_{i}}=0 \\
\frac{\partial \rho u_{i}}{\partial t}+\nabla \cdot\left(\rho u_{i} u_{j}\right)=\nabla \cdot T+\rho g_{i}+f_{\sigma i}
\end{gathered}
$$

where the subscripts $i$ and $j$ indicate the $i^{\text {th }}$ and $j^{\text {th }}$ directions of the Cartesian coordinate system respectively, $u$ is velocity, $g$ is gravitational acceleration, $t$ is time, $f_{\sigma}$ is the surface tension, and $T$ is the stress tensor which contains the pressure $P$.

Assuming that only two kinds of incompressible fluids are involved, Eq. (1) can be written as:

$$
\rho=\alpha \rho_{1}+(1-\alpha) \rho_{2} ; \quad \mu=\alpha \mu_{1}+(1-\alpha) \mu_{2}
$$

Upon applying Eq. (2)-(4), the mass conservation equation becomes: 


$$
\frac{\partial \rho}{\partial t}+u_{i} \frac{\partial \rho}{\partial x_{i}}+\rho \frac{\partial u_{i}}{\partial x_{i}}=\frac{d \rho}{d t}+\rho \nabla \cdot \vec{U}=\frac{d\left(\alpha \rho_{1}+(1-\alpha) \rho_{2}\right)}{d t}+\rho \nabla \cdot \vec{U}=0
$$

Subject to the incompressibility condition, $\nabla \cdot \vec{U}=\partial u_{i} / \partial x_{i}=0$, then,

$$
\frac{d\left(\alpha \rho_{1}+(1-\alpha) \rho_{2}\right)}{d t}=\left(\rho_{1}-\rho_{2}\right) \frac{d \alpha}{d t}=0
$$

Finally, the conservative form of the scalar advection equation for the volume fraction $\alpha$ can be written as:

$$
\begin{aligned}
\frac{d \alpha}{d t}=\frac{\partial \alpha}{\partial t}+u_{i} \frac{\partial \alpha}{\partial x_{i}}=0 & \Rightarrow \frac{\partial \alpha}{\partial t}+u_{i} \frac{\partial \alpha}{\partial x_{i}}+\alpha \frac{\partial u_{i}}{\partial x_{i}}=\frac{\partial \alpha}{\partial t}+\frac{\partial\left(u_{i} \alpha\right)}{\partial x_{i}}=0 \\
& \Rightarrow \frac{\partial \alpha}{\partial t}+\nabla \cdot(\alpha \vec{U})=0
\end{aligned}
$$

To summarize, the governing equations, including the continuity equation Eq. (2), the momentum equation Eq. (3) and the scalar transport equation Eq. (7), together with the constitutive relations given by Eq. (4), need to be solved simultaneously. In addition, the Continuum Surface Force (CSF) [39] model is adopted to calculate the surface tension force $f_{\sigma}$.

After setting up the governing equations for incompressible two-phase flows, the next step is to develop a proper space/time discretization scheme. The Pressure Implicit with Splitting of Operators (PISO) algorithm [40] is employed to deal with the pressure-velocity coupling problem on a collocated grid system. The discretization of the continuity and momentum equations follows standard practices, which are omitted here for the conciseness of the presentation. The discretization of the VOF equation, i.e. Eq. (7), is the key to accurately predicting the sharp interface between two immiscible fluids, so it is the focus in the remainder of this article.

\section{TEMPORAL DISCRETIZATION OF THE VOF EQUATION}

The VOF equation is integrated over a control volume $\mathrm{V}$ and a time interval $\delta t$ with finite volume method. If $p$ indicates the center of the control volume and $f$ represents the centroid of its boundary, upon applying Gauss' theorem, Eq. (7) leads to:

$$
\begin{gathered}
\int_{t}^{t+\delta t}\left(\int_{V} \frac{\partial \alpha}{\partial t} d V\right) d t+\int_{t}^{t+\delta t}\left(\int_{V} \nabla \cdot(\alpha \vec{U}) d V\right) d t=\int_{t}^{t+\delta t}\left(\frac{\partial \alpha}{\partial t} \cdot V_{p}\right) d t+\int_{t}^{t+\delta t}\left(\sum_{f=1}^{n} \alpha_{f} \vec{U}_{f} \cdot \vec{A}_{f}\right) d t=0 \\
\Rightarrow\left(\alpha_{p}^{t+\delta t}-\alpha_{p}^{t}\right) V_{p}+\int_{t}^{t+\delta t}\left(\sum_{f=1}^{n} \alpha_{f} \vec{U}_{f} \cdot \vec{A}_{f}\right) d t=0
\end{gathered}
$$


In order to approximate the time integral of the convection term in Eq. (8), a general strategy is to introduce a weighting parameter $\eta$ ranging between zero and one [41].

$$
\left(\alpha_{p}^{t+\delta t}-\alpha_{p}^{t}\right) V_{p}+\sum_{f=1}^{n}\left[(1-\eta) \cdot\left(\alpha_{f} \vec{U}_{f} \cdot \vec{A}_{f}\right)^{t}+\eta \cdot\left(\alpha_{f} \vec{U}_{f} \cdot \vec{A}_{f}\right)^{t+\delta t}\right] \cdot \delta t=0
$$

Obviously, $\eta=0, \eta=1$ and $\eta=1 / 2$ denote the Euler explicit, the Euler implicit and the Crank-Nicholson schemes, respectively. As is well known, the first-order implicit scheme, although computationally robust and efficient, introduces substantial numerical diffusion in the flow direction, while the first-order explicit scheme suffers from instability for a Courant number larger than 1. The second-order Crank-Nicholson scheme exhibits less diffusion, but may result in instability for a Courant number above 0.5 [5].

The original CICSAM employs the second-order Crank-Nicholson scheme in the temporal discretization. On the one hand, it is believed that, for a sufficiently small time step, the variation of volumetric flux at the cell face, defined as $F_{f}=\boldsymbol{U}_{f} \cdot \boldsymbol{A}_{f}$, is negligible in comparison with the variation of the volume fraction $\alpha_{f}$. Therefore, it is rational to simply adopt the most recent value of $F_{f}$ in Eq. (9), yielding

$$
\left(\alpha_{p}^{t+\delta t}-\alpha_{p}^{t}\right) V_{p}+\sum_{f=1}^{n}\left[F_{f} \cdot \frac{1}{2}\left(\alpha_{f}^{t}+\alpha_{f}^{t+\delta t}\right)\right] \cdot \delta t=0
$$

On the other hand, the value of $\alpha_{f}^{t}$ is calculated according to a weighting factor, $\beta_{f}$, which carries all the information about the fluid distribution and the interface orientation. The value of $\beta_{f}$ can be calculated with NVD/NVSF schemes. To obtain the value of $\alpha_{f}^{t+\delta t}$, the weighting factor at the new time is necessary, which is not available. If the time step is small enough, it is reasonable to assume that the new weighting factor can be approximated by the value at the previous time step [1,3], which is similar to the calculation of $F_{f}$. Hence,

$$
\left(\alpha_{p}^{t+\delta t}-\alpha_{p}^{t}\right) V_{p}+\sum_{f=1}^{n}\left\{F_{f} \cdot\left[\left(1-\beta_{f}\right) \cdot \frac{\alpha_{D}^{t}+\alpha_{D}^{t+\delta t}}{2}+\beta_{f} \cdot \frac{\alpha_{A}^{t}+\alpha_{A}^{t+\delta t}}{2}\right]\right\} \cdot \delta t=0
$$

where the subscripts D and A denote the donor and acceptor cells, respectively, determined by the flow direction, as indicated in Figure 1.

If a large time step is taken, the above calculations of $F_{f}$ and $\beta_{f}$ become inaccurate and unjustified. Actually, Ubbink and Issa [1] recommended that the Courant number $C_{f}$, defined as the total amount of fluid convected through the donor cell per time step divided by the volume of the donor cell, should be less than 0.3 to accurately track the free surface. In order to remove the low-Courant-number constraint, Gopala and van 
Wachem [28] proposed a so-called sub-stepping technique, where the calculation of the advection of the volume fraction is broken up into a few steps after the flow equations are solved. Furthermore, HiRAC [3] introduces a Jacobi-type dual timestepping approach, which provides an efficient way of implementing the aforementioned sub-stepping technique. However, the so-called sub-stepping technique is computationally inefficient. Therefore, the newly proposed M-CICSAM still employs the Crank-Nicholson formulation, Eq. (11), in the same way as the original CICSAM.

\section{SPATIAL DISCRETIZATION OF THE VOF EQUATION}

As can be seen in Eq. (11), the weighting factor $\beta_{f}$ is required for the discretization of the volume fraction equation. This factor is dependent on the fluid distribution and the interface orientation, and also implicitly contains the far-upwind value $\alpha_{U}$. With the aid of the NVD/NVSF concept, we obtain an effective weighting factor $\beta_{f}$ by mixing a compressive downwind scheme and a diffusive high-resolution scheme.

\subsection{The Basic Differencing Schemes of M-CICSAM}

As shown in Figure 2, the Convection Boundedness Criterion (CBC) for explicit flow calculations is widely considered to be both sufficient and necessary for the local boundedness property. The explicit CBC is defined mathematically as follows [42]:

$$
\begin{array}{ll}
\tilde{\alpha}_{D} \leq \tilde{\alpha}_{f} \leq \min \left(\tilde{\alpha}_{D} / c_{f}, 1\right) & \text { for } 0<\tilde{\alpha}_{D}<1 \\
\tilde{\alpha}_{f}=\mathrm{f}\left(\tilde{\alpha}_{D}\right)=\tilde{\alpha}_{D} & \text { for } \quad \tilde{\alpha}_{D} \geq 1, \tilde{\alpha}_{D} \leq 0
\end{array}
$$

where $c_{f}=F_{f} \cdot \delta t / V_{D}$ is the Courant number at the cell face, and the normalized variables $\tilde{\alpha}_{f}$ and $\tilde{\alpha}_{D}$ are calculated using the following expressions:

$$
\tilde{\alpha}_{f}=\frac{\alpha_{f}-\alpha_{U}}{\alpha_{A}-\alpha_{U}}, \quad \tilde{\alpha}_{D}=\frac{\alpha_{D}-\alpha_{U}}{\alpha_{A}-\alpha_{U}}
$$

According to the downwind weighting factor (DWF) method of Leonard and Mokhtari [43], the weighting factor $\beta_{f}$ can be obtained by algebraic manipulation of Eq. (11) and Eq. (13).

$$
\beta_{f}=\frac{\tilde{\alpha}_{f}-\tilde{\alpha}_{D}}{1.0-\tilde{\alpha}_{D}}
$$

CICSAM believes that the Hyper-C scheme, being the upper bound of the explicit $\mathrm{CBC}$, is the most compressive differencing scheme for the explicit discretization of 
the volume fraction equation, and is thus most suitable for the advection of a sharp interface, at least when the interface is parallel to the cell face. The Hyper-C scheme is given by:

$$
\tilde{\alpha}_{f_{\text {Hyper }-}}= \begin{cases}\min \left\{\tilde{\alpha}_{D} / c_{f}, 1\right\} & \text { when } 0 \leq \tilde{\alpha}_{D} \leq 1 \\ \tilde{\alpha}_{D} & \text { when } \tilde{\alpha}_{D}>1 \text { or } \tilde{\alpha}_{D}<0\end{cases}
$$

However, the Hyper-C scheme tends to compress any gradient into a step profile, and therefore distorts the free surface when the interface is normal to the cell face. CICSAM overcomes this problem by switching to a more diffusive high-resolution scheme. The basic non-compressive high-resolution scheme of CICSAM is the ULTIMATE-QUICKEST (UQ) of Leonard, which helps preserve the interface shape when the orientation of the interface is almost tangential to the flow direction, and can be written as $[1,42]$ :

$$
\tilde{\alpha}_{f_{U Q}}= \begin{cases}\min \left\{c_{f} \tilde{\alpha}_{D}+\left(1-c_{f}\right)\left(6 \tilde{\alpha}_{D}+3\right) / 8, \tilde{\alpha}_{f_{H y p e r}-C}\right\} & \text { when } 0 \leq \tilde{\alpha}_{D} \leq 1 \\ \tilde{\alpha}_{D} & \text { when } \tilde{\alpha}_{D}>1 \text { or } \tilde{\alpha}_{D}<0\end{cases}
$$

It should be noted that, as the Courant number $c_{f}$ increases, the abovementioned two basic differencing schemes gradually approach the very diffusive firstorder upwind scheme, and the three of them become identical at a Courant number of 1.0. Such a property undoubtedly decreases the resolution of the interface, and actually is regarded as one inherent drawback of the original CICSAM scheme. In the spirit of Leonard's ULTIMATE strategy, the explicit CBC is a result of the used temporal bounding, and is only needed for explicit transient schemes. Hence, when the Crank-Nicholson scheme is adopted for temporal discretization, it is not indispensable to employ the explicit $\mathrm{CBC}$ to achieve the local boundedness property.

Ubbink and Issa [1] point out that CICSAM can ensure acceptable numerical accuracy when the Courant number $c_{f}$ is no more than 0.3. Therefore, in the basic compressive differencing scheme $(\mathrm{CN}-\mathrm{CBC})$ of the newly proposed M-CICSAM, it is sensible to retain the Hyper-C formulation for $c_{f} \leq 0.3$. However, for large Courant numbers $\left(c_{f}>0.7\right)$, our numerical tests indicate that the SUPERBEE formulation [44], which is the basic compressive scheme of STACS [5], should be adopted, as it provides a good balance between ensuring the necessary compression and maintaining the interface shape. Furthermore, for moderate Courant numbers (e.g. $\left.0.3<c_{f} \leq 0.6\right)$, the formulation of $\tilde{\alpha}_{f}=\min \left\{\tilde{\alpha}_{D} / 0.3,1.0\right\}$ can be selected because of its excellent performance in terms of reducing numerical diffusion. 
Finally, the basic compressive differencing scheme (CN-CBC) of the newly proposed M-CICSAM scheme is defined as:

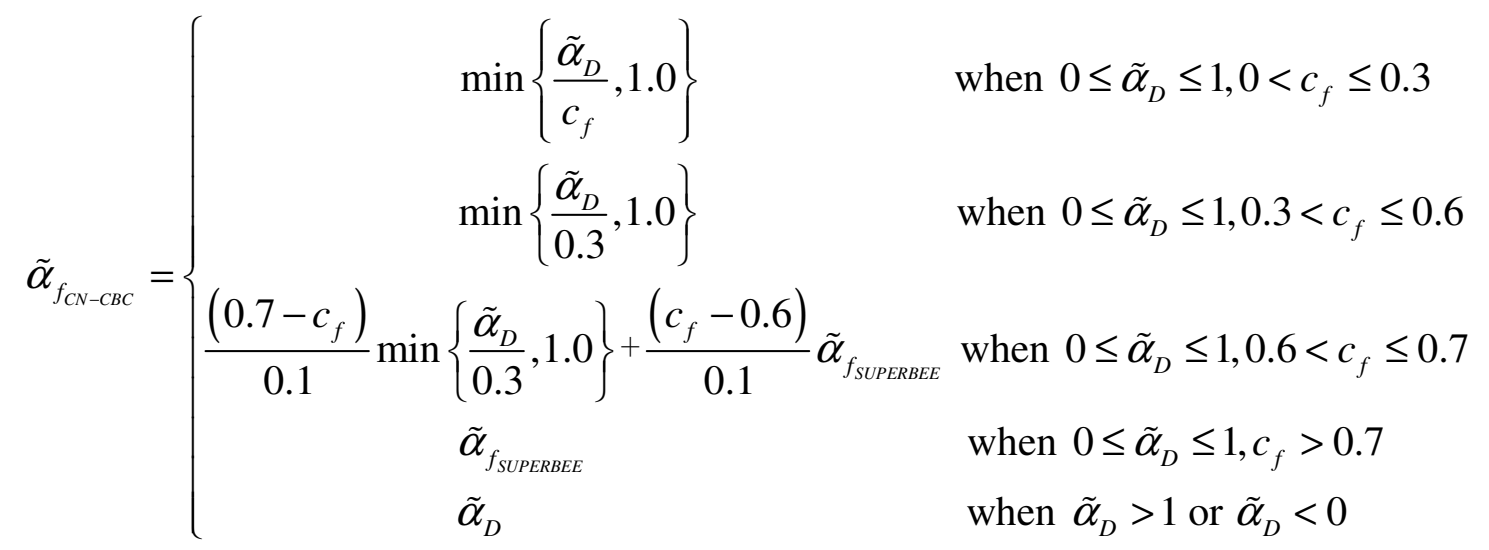

where the SUPERBEE formulation, $\tilde{\alpha}_{f_{\text {SUPERBEE }}}$, in the NVSF form [45] is described as:

$$
\tilde{\alpha}_{f_{\text {SUPEREE }}}= \begin{cases}\left(2 \tilde{x}_{f}-\tilde{x}_{D}\right) / \tilde{x}_{D} \cdot \tilde{\alpha}_{D} & \text { when } 0 \leq \tilde{\alpha}_{D}<\tilde{x}_{D} /\left(2-\tilde{x}_{D}\right) \\ \left(\tilde{x}_{D}-\tilde{x}_{f}\right) /\left(\tilde{x}_{D}-1\right)+\left(\tilde{x}_{f}-1\right) /\left(\tilde{x}_{D}-1\right) \cdot \tilde{\alpha}_{D} & \text { when } \tilde{x}_{D} /\left(2-\tilde{x}_{D}\right) \leq \tilde{\alpha}_{D}<\tilde{x}_{D} \\ \tilde{x}_{f} / \tilde{x}_{D} \cdot \tilde{\alpha}_{D} & \text { when } \tilde{x}_{D} \leq \tilde{\alpha}_{D}<\tilde{x}_{D} / \tilde{x}_{f} \\ 1 & \text { when } \tilde{x}_{D} / \tilde{x}_{f} \leq \tilde{\alpha}_{D} \leq 1 \\ \tilde{\alpha}_{D} & \text { elsewhere }\end{cases}
$$

The normalized variables $\tilde{x}_{f}$ and $\tilde{x}_{D}$ are obtained from:

$$
\tilde{x}_{f}=\frac{x_{f}-x_{U}}{x_{A}-x_{U}}, \quad \tilde{x}_{D}=\frac{x_{D}-x_{U}}{x_{A}-x_{U}}
$$

In addition, the MUSCL scheme of Van Leer [46] is employed as the basic diffusive high-resolution scheme of M-CICSAM and its mathematical formulation in the NVSF form can be written as:

$$
\tilde{\alpha}_{f_{M U S C L}}= \begin{cases}\left(2 \tilde{x}_{f}-\tilde{x}_{D}\right) / \tilde{x}_{D} \cdot \tilde{\alpha}_{D} & \text { when } 0 \leq \tilde{\alpha}_{D}<\tilde{x}_{D} / 2 \\ \tilde{x}_{f}-\tilde{x}_{D}+\tilde{\alpha}_{D} & \text { when } \tilde{x}_{D} / 2 \leq \tilde{\alpha}_{D}<1+\tilde{x}_{D}-\tilde{x}_{f} \\ 1 & \text { when } 1+\tilde{x}_{D}-\tilde{x}_{f} \leq \tilde{\alpha}_{D} \leq 1 \\ \tilde{\alpha}_{D} & \text { elsewhere }\end{cases}
$$

Obviously, the two basic differencing schemes of M-CICSAM, CN-CBC, as specified by Eq. (17)-(19), and MUSCL, as specified by Eq. (20), resolve the aforementioned deficiency associated with their counterparts in CICSAM, Hyper-C and UQ. After determining the two new basic differencing schemes, the next step is to 
develop a proper switching strategy, through which M-CICSAM smoothly changes between these basic differencing schemes, depending on the angle between the orientation of the interface and the flow direction.

\subsection{The Switching Strategy of M-CICSAM}

As mentioned earlier, the blended high-resolution schemes switch in a continuous manner between a compressive downwind scheme and a diffusive high-resolution scheme, depending on the angle between the flow direction and the grid lines. Thus, a blended face value is given by:

$$
\tilde{\alpha}_{f}=\gamma_{f} \cdot \tilde{\alpha}_{f \text {-compressive }}+\left(1-\gamma_{f}\right) \cdot \tilde{\alpha}_{f \text {-diffusive }}
$$

where the weighting function, $\gamma_{f}$, as suggested by Heyns et al. [3], is:

$$
\gamma_{f}=\min \left\{\left(\left|\frac{(\nabla \alpha)_{D} \cdot \vec{d}}{\left|(\nabla \alpha)_{D}\right| \cdot|\vec{d}|}\right|\right)^{m}, 1\right\}
$$

where $m$ is a constant introduced to control the dominance of the different schemes, $(\nabla \alpha)_{D}$ is the gradient of the volume fraction in the donor cell, and $\vec{d}$ is the distance vector connecting the centers of the donor and acceptor cells. With $m=2$, Eq. (22) reduces to the switching weighting function of the original CICSAM scheme [1]; with $m=1 / 2$ and $m=4$, it reduces to the switching weighting functions of HRIC [36] and STACS [5], respectively.

As shown in Figure 3, Eq. (22) has been developed in an Eulerian framework, which leads to physical results for one-dimensional flows. Unfortunately, with regard to multi-dimensional flow analysis, the aforementioned switching weighting function, derived from one-dimensional configuration, cannot always guarantee physically sensible results, which is deemed to be another inherent drawback of the classic blended high-resolution schemes put forward in the past decades.

As illustrated in Figure 4, we consider the advection of an interface in a two-dimensional (2D) uniform velocity field, where the flow is in the diagonal direction and the Courant number is 0.5 . In this condition, the angle between the interface orientation $\left((\nabla \alpha)_{D} /\left|(\nabla \alpha)_{D}\right|\right)$ and the line connecting the centers of the donor and acceptor cells $(\vec{d})$ is 45 degrees. According to Eq. (22), neither the compressive downwind scheme nor the diffusive high-resolution scheme should be utilized at this angle. However, it is actually reasonable to adopt a compressive downwind scheme at the highlighted cell face $E F$, which can be revealed from the 
exact solution of this 2D problem and thus demonstrates the deficiency of Eq. (22) in the two-dimensional situation.

Therefore, a more reasonable switching algorithm is needed to achieve physically sound results irrespective of the dimensionality of the flows and the topological structure of computational meshes. Due to the complexity of three-dimensional (3D) unstructured meshes, we start by considering $2 \mathrm{D}$ structured meshes and then extend the newly proposed 2D switching strategy to 3D problems.

The key to a successful 2D switching strategy is the inclusion of the velocity vector $\vec{V}$ at the cell face in its formulation. Obviously, the angle $\left(\theta_{f-1}\right)$ between the velocity vector $\vec{V}$ and the unit vector $\vec{n}$ outward normal to the cell face plays an important role in determining how to switch, but it is equally important to take into account the influences of the angle $\left(\theta_{f-2}\right)$ between the interface orientation $\left((\nabla \alpha)_{D} /\left|(\nabla \alpha)_{D}\right|\right)$ and the velocity vector $\vec{V}$.

Taking $\theta_{f-1}=45^{\circ}$, positive in the clockwise direction, as an example, Figures 5-8 demonstrate the accurate flux crossing a specified donor-cell face ( $E F$ ) during one time step at a Courant number of 0.5 with some representative values of $\theta_{f-2}$, with the aim to design a sensible 2D switching strategy for M-CICSAM. As displayed in Figure $8\left(\theta_{f-2}=135^{\circ}\right.$ and $\left.\theta_{f-2}=315^{\circ}\right)$, when the interface orientation is parallel to the cell face, only fluid present at the downstream cell should be convected through the cell face, which is in accordance with the original 1D switching strategy. Special attention should be paid to Figure $7\left(\theta_{f-2}=90^{\circ}\right.$ and $\left.\theta_{f-2}=270^{\circ}\right)$, where the interface orientation is neither parallel nor perpendicular to the cell face. According to Eq. (22), the value of $\tilde{\alpha}_{f}$ should be obtained by blending the compressive downwind and diffusive high-resolution schemes. However, by examining the exact fluxes of the volume fraction across $E F$, it can be concluded that the adoption of Eq. (22) is not advisable and the compressive downwind scheme is most suitable under this circumstance.

Similarly, Figures 9-12 and Figures 13-16, in which the angle $\theta_{f-1}$ is equal to $0^{\circ}$ and $-45^{\circ}$, respectively, describe the exact fluxes of the volume fraction across $E F$ at different $\theta_{f-2}$ values. When it comes to $\theta_{f-1}=90^{\circ}$ and $\theta_{f-1}=-90^{\circ}$, with the velocity vector $\vec{V}$ perpendicular to the unit vector $\vec{n}$, the projection of $\vec{V}$ on to the unit vector $\vec{n}$ becomes zero, so no fluid can pass through the cell face. In theory, any value of $\tilde{\alpha}_{f}$ is appropriate in this case. Nonetheless, numerical tests show that the compressive downwind scheme gives the best results. For different combinations 
of $\theta_{f-1}$ and $\theta_{f-2}$, it is necessary to employ different switching functions to blend the aforementioned two basic differencing schemes, in order to obtain the precise fluxes of the volume fraction across $E F$, which are shown as the cross-hatched regions in these figures.

In Table 1, the third column demonstrates the recommended switching function $\gamma_{f-2-u}$ or $\gamma_{f-2-l}$ for each combination, which will be defined in detail in the following and is dependent on the bounds of $\theta_{f-1}$ and the ranges of $\theta_{f-2}$, listed in the first two columns. In summary, the new 2D switching strategy proposed herein can be written as:

$$
\tilde{\alpha}_{f}=\gamma_{f-1} \cdot \tilde{\alpha}_{\left(\theta_{f-1}\right)^{\text {lower-bound }}}+\left(1-\gamma_{f-1}\right) \cdot \tilde{\alpha}_{\left(\theta_{f-1}\right)^{\text {upper-bound }}}
$$

where

$$
\begin{aligned}
& \gamma_{f-1}= \begin{cases}{\left[\cos \left(2\left|\theta_{f-1}\right|\right)\right]^{2}} & \text { if } \theta_{f-1} \in\left[-90^{\circ},-45^{\circ}\right] \\
{\left[\sin \left(2\left|\theta_{f-1}\right|\right)\right]^{2}} & \text { if } \theta_{f-1} \in\left[-45^{\circ},-0^{\circ}\right] \\
{\left[\cos \left(2 \theta_{f-1}\right)\right]^{2}} & \text { if } \theta_{f-1} \in\left[0^{\circ}, 45^{\circ}\right] \\
{\left[\sin \left(2 \theta_{f-1}\right)\right]^{2}} & \text { if } \theta_{f-1} \in\left[45^{\circ}, 90^{\circ}\right]\end{cases} \\
& \theta_{f-1}= \begin{cases}\arccos \mid \frac{\vec{V} \cdot \vec{n}}{|\vec{V}| \cdot|\vec{n}|} & \text { if } \theta_{f-1} \text { is in the clockwise direction } \\
-\arccos \mid \frac{\vec{V} \cdot \vec{n}}{|\vec{V}| \cdot|\vec{n}|} & \text { if } \theta_{f-1} \text { is in the anti-clockwise direction }\end{cases} \\
& \tilde{\alpha}_{\left(\theta_{f-1}\right)}^{\text {upper-bound }}=\gamma_{f-2-u} \cdot \tilde{\alpha}_{f_{C N-C B C}}+\left(1-\gamma_{f-2-u}\right) \cdot \tilde{\alpha}_{f_{M U S C L}} \\
& \tilde{\alpha}_{\left(\theta_{f-1}\right)^{\text {lower-bound }}}=\gamma_{f-2-l} \cdot \tilde{\alpha}_{f_{C N-C B C}}+\left(1-\gamma_{f-2-l}\right) \cdot \tilde{\alpha}_{f_{M U S C L}}
\end{aligned}
$$

where $\gamma_{f-2-u}$ is a weighting function determined by both $\left(\theta_{f-1}\right)^{u p p e r}$, the upper bound of $\theta_{f-1}$, and $\theta_{f-2}$. Similarly, $\gamma_{f-2-l}$ is a weighting function decided by both $\left(\theta_{f-1}\right)^{\text {lower }}$, the lower bound of $\theta_{f-1}$, and $\theta_{f-2}$.

In principle, as demonstrated in Eq. (23)-(25), for an arbitrary $\theta_{f-1}$ value, with the aid of the weighting function $\gamma_{f-1}$, we are able to obtain the value of $\tilde{\alpha}_{f}$ by blending two extreme values, the values for the lower and upper bounds of $\theta_{f-1}$ $\tilde{\alpha}_{\left(\theta_{f-1}\right)^{\text {lower-bound }}}$ and $\tilde{\alpha}_{\left(\theta_{f-1}\right)}$ upper-bound $)$, which can be exactly calculated either by the compressive scheme (CN-CBC) or by the diffusive high-resolution scheme (MUSCL) for typical $\theta_{f-2}$ values, as displayed in Figures 4-16. However, in practical situations, 
$\theta_{f-2}$ may not be equal to the typical values depicted in these Figures. Therefore, with regard to a specific bound of $\theta_{f-1}$ and an arbitrary $\theta_{f-2}$ value, the weighting function $\gamma_{f-2-u}$ or $\gamma_{f-2-l}$ is introduced to blend the aforementioned two basic differencing schemes, as described in Eq. (26)-(27).

In practical applications, we first confirm the values of $\left(\theta_{f-1}\right)^{\text {upper }}$ and $\left(\theta_{f-1}\right)^{\text {lower }}$ according to the first column of Table 1 , then, based on the range of $\theta_{f-2}$ provided in the second column, we select the corresponding expressions for $\gamma_{f-2-u}$ and $\gamma_{f-2-l}$ from the third column.

\subsection{The Far-upwind Reconstruction on Unstructured Meshes}

As can be seen in Eq. (13) and Eq. (19), the far-upwind node location $x_{U}$ and variable value $\alpha_{U}$ are required in the calculation of the normalized variable $\tilde{\alpha}_{f}$. However, on arbitrary unstructured grids, the far-upwind node $U$ is not readily available and therefore the aforementioned blended high-resolution schemes are no longer straightforward in their implementation.

The existing technique commonly found in the literature resolves this issue by constructing a virtual far-upwind node using equal-distance and equal-gradient assumptions [47]. In this way, it is supposed that the distance between the upwind cell $U$ and the donor cell $D, d u$, is equal to that between $D$ and the acceptor cell $A, d d$. It furthermore assumes that the volume fraction $\alpha$ uniformly changes from $U$ to $A$, so the following formula can be derived [1]:

$$
(\nabla \alpha)_{D} \cdot \vec{d}(d u+d d)=(\nabla \alpha)_{D} \cdot \vec{d} \cdot 2 d d=\alpha_{A}-\alpha_{U}^{*}
$$

Finally,

$$
\alpha_{U}^{*}=\alpha_{A}-(\nabla \alpha)_{D} \cdot \vec{d} \cdot 2 d d
$$

where $\vec{d}$ is the unit vector from $D$ to $A$. To guarantee $\alpha_{U}^{*}$ is physically bounded, it is necessary to restrict this value within the maximum and minimum variable values, $\alpha_{D}^{\max }$ and $\alpha_{D}^{\min }$, at the donor cell's nearest neighbors.

$$
\alpha_{U}^{*}=\min \left\{\max \left\{\alpha_{U}^{*}, \alpha_{D}^{\min }\right\}, \alpha_{D}^{\max }\right\}
$$

Zhang et al. [48] argued that this original reconstruction technique has two inherent drawbacks. Firstly, the imaginary node $U$ may be located several cells away from the donor cell $D$, when extremely non-uniform grids are deployed. Secondly, it is inappropriate to assume that the gradient $\nabla \alpha$ does not change along the whole segment $U A$. In order to overcome the aforementioned two drawbacks, 
New-Technique-2 is put forward in their paper, where a predictor-corrector procedure is undertaken to determine the value of $d u$.

As shown in a non-orthogonal mesh in Figure 17, for the interface ab between the donor cell $D$ and the acceptor cell $A$, the face variable value $\alpha_{f}$ is calculated using auxiliary nodes $D^{\prime}$ and $A^{\prime}$, which are the projections of $D$ and $A$, respectively, along the normal direction of the face. The distance between $D^{\prime}$ and $A^{\prime}$ is defined as $d d$. In the diffusion term discretization, the differences between the physical quantities at $D^{\prime}$ and $D$ (or $A^{\prime}$ and $A$ ) can be approximated by explicit non-orthogonal correction. In the convection term discretization, however, the above-mentioned differences are neglected, which means that the node $D^{\prime}$ (or $A^{\prime}$ ) has the same values and gradients as the node $D$ (or $A$ ), i.e., $\alpha_{D}=\alpha_{D^{\prime}}, \alpha_{A}=\alpha_{A^{\prime}}$, $(\nabla \alpha)_{D}=(\nabla \alpha)_{D^{\prime}}$ and $(\nabla \alpha)_{A}=(\nabla \alpha)_{A^{\prime}}$.

All the donor-cell's nodes are taken into account except for the two end nodes of the chosen face $(\mathrm{a}, \mathrm{b})$. A vector quantity from each node to $D$ is calculated, such as $\overrightarrow{c D}, \overrightarrow{d D}, \overrightarrow{e D}$ and $\overrightarrow{f D}$, depicted as the dashed lines in Figure 17. If the dot product of a vector with the unit outward normal vector $\vec{n}$ of the chosen face has a positive value, then this vector's projection along the outward normal direction is recorded, such as $\overrightarrow{d^{\prime} D}, \overrightarrow{e^{\prime} D}$ and $\overrightarrow{f^{\prime} D}$ for the case of Figure 17. Finally, the average of these projections is computed to determine the predictor value of $d u$. In general,

$$
d u_{\text {predictor }}=\operatorname{Average}\left(N_{1}^{\prime} D+N_{2}^{\prime} D+N_{3}^{\prime} D+\cdots \cdots+N_{n}^{\prime} D\right)
$$

where $N_{1}^{\prime} D, N_{2}^{\prime} D, \cdots \cdots$ and $N_{n}^{\prime} D$ are the magnitudes of the projections, such as $\overrightarrow{d^{\prime} D}, \overrightarrow{e^{\prime} D}$ and $\overrightarrow{f^{\prime} D}$ in the case of Figure 17, whose dot products with the unit outward normal vector $\vec{n}$ of the face are positive. Then, in the corrector step,

$$
d u=\min \left(d u_{\text {original }}, d u_{\text {predictor }}\right)
$$

where $d u_{\text {predictor }}$ and $d u_{\text {original }}$ are the distances calculated in the predictor step and the original reconstruction technique, respectively, as shown in Figure 18.

Moreover, in New-Technique-2, a parabolic function is used to represent the volume fraction variation over the interval $\left[x_{U}, x_{D}\right]$, based on $\alpha_{D},(\nabla \alpha)_{D}$ and $\alpha_{A}$. Mathematically,

$$
\alpha_{U}^{*}=\frac{\alpha_{A}-\alpha_{D}-(\nabla \alpha)_{D} \times d d}{d d^{2}} d u^{2}+(\nabla \alpha)_{D} \times(-d u)+\alpha_{D}
$$

For the purpose of ensuring the physical boundedness of $\alpha_{U}^{*}$, Eq. (30) is also adopted to restrict the value of $\alpha_{U}^{*}$, and the bounds are set to be the maximum and 
minimum values of the donor cell $D$ and all its neighbor cells except for the acceptor cell $A$. Reference [48] clearly shows that New-Technique-2 results in a better performance in terms of overall accuracy and convergence, when compared with the original reconstruction technique. In total, eleven classic NVSF schemes were tested on arbitrary unstructured meshes.

Therefore, for M-CICSAM, New-Technique-2, described by Eqs. 30-33, is selected as the far-upwind reconstruction technique on arbitrary unstructured meshes. Then, the formulation of the new VOF interface capturing scheme (M-CICSAM) have been completed. The implementation of M-CICSAM is straightforward, because the value of $\alpha_{f}$ can be calculated in the same way for all the faces irrespective of the dimensionality of control volumes and the topology of grids.

In summary, M-CICSAM overcomes three inherent drawbacks of the previous blended high-resolution schemes by employing two new basic NVSF differencing schemes (Eqs. 17-20), a novel 2D switching strategy (Eqs. 23-27 and Table 1), and an improved far-upwind reconstruction technique (Eqs. 30-33). In the next section, the relative performance of different blended high-resolution schemes is examined by various numerical tests.

\section{NUMERICAL TEST CASES}

This section presents several classic numerical examples to evaluate the relative performance of five blended high-resolution interface capturing schemes, including CICSAM, HRIC, STACS, THOR and M-CICSAM.

Prescribed velocity fields are used and no attempt is made to consider the coupling between the volume fraction and momentum equations. For the purpose of comparison, the average error is defined as:

$$
E=\frac{1}{N} \sum_{i}^{N}\left\|\alpha_{i}^{n}-\alpha_{i}^{a}\right\|
$$

where $\alpha_{i}^{n}$ is the calculated solution after $n$ time steps, $\alpha_{i}^{a}$ the exact analytical solution and $N$ the number of control volumes [1,3].

5.1. Test 1: Advection of a square droplet in a uniform velocity field

In this problem, we consider the advection of a $0.3 \times 0.3$ square droplet in an oblique uniform velocity field of $(u, v)=(2,1)$. As shown in Figure 19, the computational 
domain is a square of side length 1.2, and the square droplet is initially centered at $(0.3,0.3)$, with its exact position centered at $(0.9,0.6)$ after $0.3 \mathrm{~s}$. A uniformly spaced mesh of $120 \times 120$ cells is employed in this test, as presented in Figure 20 . Computations are performed at seven different time steps, yielding Courant numbers of $0.2,0.3,0.4,0.5,0.6,0.7$ and 0.8 , respectively.

Table 2 summarizes the errors of various blending high-resolution schemes at different Courant numbers and Figure 21 gives a graphical representation of these errors. Obviously, when compared with the other four schemes, M-CICSAM leads to more accurate solutions, especially at high Courant numbers. Figures 22-26 demonstrate the contour plots of the volume fraction for different schemes at a Courant number of 0.6 at the end of the computation. As displayed in Figures 22-24, HRIC, THOR and CICSAM introduce unacceptable levels of numerical diffusion even at a moderate Courant number, because the basic compressive schemes in these algorithms approach the first-order upwind scheme with increasing Courant number $[1,36]$.

Special attention needs to be paid to the STACS scheme [5], which appears to be more effective than HRIC, THOR and CICSAM in terms of preserving interface sharpness at Courant numbers above 0.5, as displayed in Table 2 and Figures 21-25. Moreover, Figure 21 seems to show that the performance of STACS is almost independent of the Courant number in this test. However, Figure 21 indicates that STACS tends to introduce more numerical diffusion when compared with CICSAM, HRIC, THOR and M-CICSAM at Courant numbers less than 0.3. The aforementioned behavior of STACS can be mainly attributed to the adoption of the famous SUPERBEE scheme, which is very compressive, but relatively more diffusive than the Hyper-C scheme of CICSAM at small Courant numbers [1, 5].

The newly proposed M-CICSAM is able to most effectively maintain the interface sharpness and preserve the interface shape at all Courant numbers, showing its apparent superiority over the remaining four schemes.

\subsection{Test 2: Advection of a round droplet in a uniform velocity field}

As illustrated in Figure 27, the same physical domain and velocity field as the square droplet case are adopted in conducting the second test, in which a circle with a diameter of 0.3 is initially centered at $(0.3,0.3)$, with its exact position centered at $(0.9,0.6)$ after 0.3 second. In this test, an unstructured mesh composed of 14678 cells, instead of the aforementioned uniform rectangular mesh of $120 \times 120$ cells, is 
employed, as depicted in Figure 28. Since it is hard to specify the Courant number over unstructured meshes, the same seven time steps as the first test are used in performing the computations, resulting in relatively higher maximum Courant numbers on the unstructured grid.

The average errors of various blended high-resolution schemes at different Courant numbers are demonstrated in Table 3 and Figure 29. Furthermore, Figures 30-34 present the contour plots of the volume fraction for different schemes at a Courant number of 0.6 after the lapse of 0.3 s. As expected, almost the same conclusions as the first test can be deduced from the numerical results, and M-CICSAM is more accurate in terms of maintaining the interface shape and preserving the interface sharpness, especially at high Courant numbers.

\subsection{Test 3: Advection of a slotted circle in a rotational flow field}

In the third test, as illustrated in Figure 35, we consider the rotation of a slotted circle with a radius of 0.3 , and a slot that is 0.12 wide and 0.3 long. The slotted circle is initially centered at $(0.35,0.35)$, and the rotational velocity field is given by:

$$
U=-\Omega \times\left(y-y_{0}\right), \quad V=\Omega \times\left(x-x_{0}\right)
$$

where $\left(x_{0}, y_{0}\right)=(0.35,0.35)$ is the center of rotation and $\Omega=0.5$ is the angular speed. Computation has been carried out on an unstructured mesh consisting of 10208 control volumes, whose resolution is comparable to a uniformly spaced mesh with $100 \times 100$ cells, as shown in Figure 36 . The problem is solved with seven different time steps, yielding Courant numbers of 0.2, 0.3, 0.4, 0.5, 0.6, 0.7 and 0.8, respectively, based on the grid size of the corresponding structured mesh.

The average errors after one revolution are summarized in Table 4, and a graphical representation of these errors is given by Figure 37. As with previous tests, predictions generated by different schemes at a Courant number of 0.5 are presented in Figures 38-42 in the form of contour plots of the volume fraction. As expected, with increasing Courant number, HRIC, THOR and CICSAM lead to much more diffusive predictions. Again, STACS introduces more numerical diffusion at small Courant numbers, when compared with HRIC, THOR, CICSAM and M-CICSAM, as evidenced in Figure 37. M-CICSAM performs better than the other four interface capturing schemes by more effectively preserving the interface sharpness and shape at all Courant numbers, as displayed in Figure 37 and Figure 42. 


\subsection{Test 4: Advection of a circle in a shear flow field}

The former three tests show the superiority of M-CICSAM when the flow field is either uniform or rotational. In those cases, the fluid moves as a rigid body and thus the computation does not involve any topological change of the interface. However, in most real interfacial flow cases, the situation is far more complicated and the initial shape is not preserved [1, 3, 28]. With the purpose of evaluating the ability of different schemes to handle shearing and stretching of the interface, the fourth test involves the advection of a circle, with radius $0.2 \pi$ and center $(0.5 \pi, 0.2(1+\pi))$, in a shear flow field, as described in Figure 43. The velocity field is given by:

$$
U=\sin (x) \cdot \cos (y), \quad V=-\cos (x) \cdot \sin (y)
$$

where $x, y \in[0, \pi]$. As shown in Figure 44, calculations are carried out on a nonuniform unstructured mesh consisting of 25636 control volumes, which is comparable to a uniformly spaced mesh of $160 \times 160$ cells. Like the third test, computation is performed with seven different time steps, yielding Courant numbers of 0.2, 0.3, 0.4, $0.5,0.6,0.7$ and 0.8 , respectively, based on the grid size of the corresponding structured mesh. The simulation is firstly run for $N=1000$ time steps, then the flow is reversed with an additional $N=1000$ time steps of simulation. Hence, the interface should return to its initial shape at the end of the simulation.

Table 5 presents the average errors of different schemes, and Figure 45 gives a graphical representation of these errors. Figures 46-50 display the contour plots of the volume fraction after 1000 and 2000 time steps of simulations at a Courant number of 0.5. Obviously, HRIC, THOR and CICSAM lead to unacceptable levels of numerical diffusion at high Courant numbers, as shown in Figures 45-48.

Compared to the aforementioned three schemes, STACS leads to more accurate numerical predictions at Courant numbers above 0.5, as evidenced in Table 5 and Figure 45. However, as noticed before, STACS introduces more numerical diffusion at Courant numbers less than 0.3.

Even in this more challenging situation, as demonstrated in Table 5 and Figures 45-50, M-CICSAM still predicts the best profiles at all Courant numbers, which is in accordance with the conclusion drawn from the previous tests and verifies its advantage once again.

\section{CONCLUSIONS}


A refined VOF interface capturing scheme, denoted as M-CICSAM, is developed in this paper. Essentially, this modified CICSAM combines two new basic differencing schemes, adopts a novel 2D switching strategy, and uses an improved far-upwind reconstruction technique suitable for arbitrary unstructured meshes. By theoretical analysis, it is concluded that M-CICSAM overcomes three inherent drawbacks of the original CICSAM. Four representative blended high-resolution schemes, including HRIC, STACS, THOR and CICSAM, are chosen to compare with M-CICSAM by solving four pure advection problems. The numerical results clearly show that M-CICSAM leads to more accurate predictions, especially at high Courant numbers, as it maintains the sharpest edges and preserves the best interface shapes in all the tests considered.

\section{ACKNOWLEDGEMENTS}

The authors gratefully acknowledge the financial support provided by the National Natural Science Foundation of China (Grant No. 51279082) and the State Key Laboratory of Hydroscience and Engineering of Tsinghua University (Grant No. 2013-KY-3). 


\section{REFERENCES}

1. Ubbink O, Issa RI. A method for capturing sharp fluid interfaces on arbitrary meshes. Journal of Computational Physics 1999; 153(1): 26-50.

2. Pericleous KA, Chan KS, Cross M. Free surface flow and heat transfer in cavities: The SEA algorithm. Numerical Heat Transfer 1995; 27(4): 487-507.

3. Heyns JA, Malan AG, Harms TM, Oxtoby OF. Development of a compressive surface capturing formulation for modelling free-surface flow by using the volume-of-fluid approach. International Journal for Numerical Methods in Fluids 2013; 71(6): 788-804.

4. Pan D, Chang $\mathrm{CH}$. The capturing of free surfaces in incompressible multi-fluid flows. International Journal for Numerical Methods in Fluids 2000; 33(2): 203-222.

5. Darwish M, Moukalled F. Convective schemes for capturing interfaces of free-surface flows on unstructured grids. Numerical Heat Transfer 2006; 49(1): 19-42.

6. Kelecy FJ, Pletcher RH. The development of a free surface capturing approach for multidimensional free surface flows in closed containers. Journal of Computational Physics 1997; 138(2): 939-980.

7. Jahanbakhsh E, Panahi R, Seif MS. Numerical simulation of three-dimensional interfacial flows. International Journal of Numerical Methods for Heat \& Fluid Flow 2007; 17(4): 384-404.

8. Unverdi SO, Tryggvason G. A front-tracking method for viscous, incompressible, multifluid flows. Journal of Computational Physics 1992; 100(1): 25-37.

9. Okamoto T, Kawahara M. Two-dimensional sloshing analysis by Lagrangian finite element method. International Journal for Numerical Methods in Fluids 1990; 11(5): $453-477$.

10. de Sousa FS, Mangiavacchi N, Nonato LG, Castelo A, Tomé MF, Ferreira VG, Cuminato JA, McKee S. A front-tracking/front-capturing method for the simulation of 3D multifluid flows with free surfaces. Journal of Computational Physics 2004; 198(2): 469-499.

11. Kim MS, Lee WI. A new VOF-based numerical scheme for the simulation of fluid flow with free surface. Part I: New free surface-tracking algorithm and its verification. International Journal for Numerical Methods in Fluids 2003; 42(7): 765-790.

12. Kothe DB, Mjolsness RC. RIPPLE: A new model for incompressible flows with free surfaces. AIAA Journal 1992; 30(11): 2694-2700.

13. Tryggvason G, Bunner B, Esmaeeli A, Juric D, Al-Rawahi N, Tauber W, Han J, Nas S, Jan Y.-J. A front-tracking method for the computations of multiphase flow. Journal of Computational Physics 2001; 169(2): 708-759. 
14. Harlow FH, Welch JE. Numerical calculation of time-dependent viscous incompressible flow of fluid with free surface. Physics of Fluids 1965; 8: 2182-2189.

15. Chan RKC, Street RL. A computer study of finite-amplitude water waves. Journal of Computational Physics 1970; 6(1): 68-94.

16. Nakayama T, Mori M. An Eulerian finite element method for time-dependent free surface problems in hydrodynamics. International Journal for Numerical Methods in Fluids 1996; 22(3): 175-194.

17. Scardovelli R, Zaleski S. Direct numerical simulation of free-surface and interfacial flow. Annual Review of Fluid Mechanics 1999; 31: 567-603.

18. Pilliod Jr JE, Puckett EG. Second-order accurate volume-of-fluid algorithms for tracking material interfaces. Journal of Computational Physics 2004; 199(2): 465-502.

19. Rudman M. A volume-tracking method for incompressible multi-fluid flows with large density variations. International Journal for Numerical Methods in Fluids 1998; 28: $357-378$

20. Meier M, Yadigaroglu G, Smith BL. A novel technique for including surface tension in PLIC-VOF methods. European Journal of Mechanics - B/Fluids 2002; 21(1): 61-73.

21. Rider WJ, Kothe DB. Reconstructing volume tracking. Journal of Computational Physics 1998; 141(2): 112-152.

22. Qian L, Causon DM, Mingham CG, Ingram DM. A free-surface capturing method for two fluid flows with moving bodies. Proceedings of the Royal Society: A 2006; 462(2065): $21-42$.

23. Sussman M, Puckett EG. A coupled level set and volume-of-fluid method for computing 3D and axisymmetric incompressible two-phase flows. Journal of Computational Physics 2000; 162(2): 301-337.

24. van der Pijl SP, Segal A, Vuik C, Wesseling P. A mass-conserving level-set method for modeling of multi-phase flows. International Journal for Numerical Methods in Fluids 2005; 47(4): 339-361.

25. LeVeque RJ. High-resolution conservative algorithms for advection in incompressible flow. SIAM Journal on Numerical Analysis 1996; 33(2): 627-665.

26. Bonometti T, Magnaudet J. An interface-capturing method for incompressible two-phase flows. Validation and application to bubble dynamics. International Journal of Multiphase Flow 2007; 33(2): 109-133.

27. Zalesak, ST. Fully multidimensional Flux-Corrected Transport algorithms for fluids. Journal of Computational Physics 1979; 31(3): 335-362. 
28. Gopala VR, van Wachem BGM. Volume of fluid methods for immiscible-fluid and free-surface flows. Chemical Engineering Journal 2008; 141(1-3): 204-221.

29. Rusche H, Computational fluid dynamics of dispersed two-phase flows at high phase fractions. Ph.D. Thesis, Imperial College of Science, Technology and Medicine, 2002.

30. Walters DK, Wolgemuth NM. A new interface-capturing discretization scheme for numerical solution of the volume fraction equation in two-phase flows. International Journal for Numerical Methods in Fluids 2009; 60(8): 893-918.

31. Thuburn J. Multidimensional flux-limited advection schemes. Journal of Computational Physics 1996; 123(1): 74-83.

32. Dendy ED, Padial-Collins NT, VanderHeyden WB. A general-purpose finite-volume advection scheme for continuous and discontinuous fields on unstructured grids. Journal of Computational Physics 2002; 180(2): 559-583.

33. Xiao F, Ikebata A. An efficient method for capturing free boundaries in multi-fluid simulations. International Journal for Numerical Methods in Fluids 2003; 42(2): 187-210.

34. Yokoi K. Efficient implementation of THINC scheme: A simple and practical smoothed VOF algorithm. Journal of Computational Physics 2007; 226(2): 1985-2002.

35. Lafaurie B, Nardone C, Scardovelli R, Zaleski S, Zanetti G. Modelling merging and fragmentation in multiphase flows with SURFER. Journal of Computational Physics 1994; 113(1): 134-147.

36. Muzaferija S, Peric M, Sames P, Schellin T. A two-fluid Navier-Stokes solver to simulate water entry. Proceedings of the Twenty-Second Symposium on Naval Hydrodynamics 1999; 638-649.

37. Tsui YY, Lin SW, Cheng TT, Wu TC. Flux-blending schemes for interface capture in two-fluid flows. International Journal of Heat and Mass Transfer 2009; 52(23-24): $5547-5556$.

38. Hogg PW, Gu XJ, Emerson DR. An implicit algorithm for capturing sharp fluid interfaces in the volume of fluid advection method. Council for the Central Laboratory of the Research Councils 2006.

39. Brackbill JU, Kothe DB, Zemach C. A continuum method for modelling surface tension. Journal of Computational Physics 1992; 100(2): 335-354.

40. Issa RI. Solution of the implicitly discretised fluid flow equations by operator-splitting. Journal of Computational Physics 1986; 62(1): 40-65.

41. Panahi R, Jahanbakhsh E, Seif MS. Comparison of interface capturing methods in two 
phase flow. Iranian Journal of Science \& Technology 2005; 29(B6): 539-548.

42. Leonard BP. The ULTIMATE conservative difference scheme applied to unsteady one-dimensional advection. Computer Methods in Applied Mechanics and Engineering 1991; 88(1): 17-74.

43. Leonard BP, Mokhtari S. Beyond first-order upwinding: the ultra-sharp alternative for non-oscillatory steady-state simulation of convection. International Journal for Numerical Methods in Engineering 1990; 30(4): 729-766.

44. Roe PL. Characteristic-based schemes for the Euler equations. Annual Review of Fluid Mechanics 1986; 18: 337-365.

45. Darwish MS, Moukalled FH. Normalized variable and space formulation methodology for high-resolution schemes. Numerical Heat Transfer 1994; 26(1): 79-96.

46. van Leer B. Towards the ultimate conservative difference scheme. V. A second-order sequel to Godunov's method. Journal of Computational Physics 1979; 32(1): 101-136.

47. Jasak H, Weller HG, Gosman AD. High resolution NVD differencing scheme for arbitrarily unstructured meshes. International Journal for Numerical Methods in Fluids 1999; 31(2): 431-449.

48. Zhang D, Jiang C, Yang C, Yang Y. Assessment of different reconstruction techniques for implementing the NVSF schemes on unstructured meshes. International Journal for Numerical Methods in Fluids 2014; 74(3): 189-221. 
Table 1. The switching weighting functions $\gamma_{f-2-u}$ and $\gamma_{f-2-l}$ for different combinations of $\theta_{f-1}$ and $\theta_{f-2}$ in the new 2D switching strategy of M-CICSAM

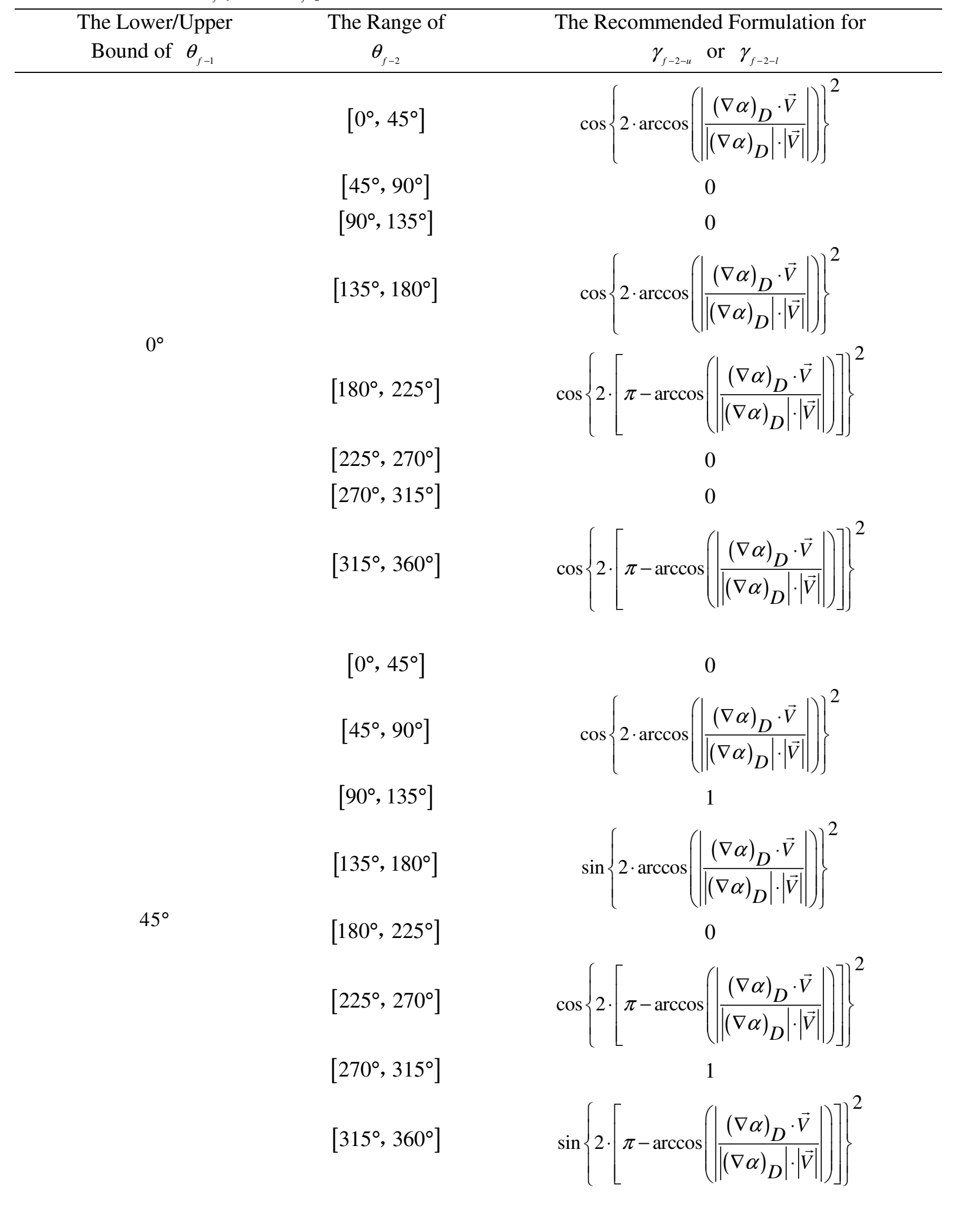


Table 1. (Continued)

\begin{tabular}{|c|c|c|c|c|}
\hline $\begin{array}{l}\text { The Lower/Upper } \\
\text { Bound of } \theta_{f-1}\end{array}$ & $\begin{array}{c}\text { The Range of } \\
\theta_{f-2} \\
\end{array}$ & The $\mathrm{Re}$ & $\begin{array}{c}\text { ecommend } \\
\gamma_{f-2-u} \\
\end{array}$ & $\begin{array}{l}\text { led Formulation for } \\
\text { or } \gamma_{f-2-l}\end{array}$ \\
\hline \multirow{11}{*}{$-45^{\circ}$} & {$\left[\begin{array}{ll}0^{\circ} & 45^{\circ}\end{array}\right]$} & \multirow{2}{*}{$\sin \{$} & \multirow{2}{*}{$2 \cdot \arccos$} & $\left.(\nabla \alpha)_{D} \cdot \vec{V} \mid\right)\left.\right|^{2}$ \\
\hline & {$\left[\begin{array}{lll}0 & 0\end{array}\right]$} & & & $\|(\nabla \alpha)_{D}|\cdot| \vec{V}|| \mid$ \\
\hline & {$\left[45^{\circ}, 90^{\circ}\right]$} & \multicolumn{3}{|c|}{1} \\
\hline & {$\left[90^{\circ}, 135^{\circ}\right]$} & \multirow{2}{*}{$\cos \{$} & \multirow{2}{*}{$2 \cdot \arccos$} & $\left.(\nabla \alpha)_{D} \cdot \vec{V} \mid\right)\left.\right|^{2}$ \\
\hline & & & & $\left\|(\nabla \alpha)_{D}|\cdot| \vec{V}\right\|$ \\
\hline & {$\left[135^{\circ}, 180^{\circ}\right]$} & \multicolumn{3}{|c|}{0} \\
\hline & {$\left[180^{\circ}, 225^{\circ}\right]$} & $\sin \{2$ & $\pi-\arccos$ & $(\nabla \alpha)_{D} \cdot \vec{V}$ \\
\hline & {$\left[225^{\circ}, 270^{\circ}\right]$} & \multicolumn{3}{|r|}{1} \\
\hline & {$\left[270^{\circ}, 315^{\circ}\right]$} & \multirow{2}{*}{$\cos \{2$. } & \multirow{2}{*}{$\pi-\arccos$} & $\|(\nabla \alpha)_{D} \cdot \vec{V}$ \\
\hline & & & & $\|\left(\left\|(\nabla \alpha)_{D}|\cdot| \vec{V}\right\|\right.$ \\
\hline & {$\left[315^{\circ}, 360^{\circ}\right]$} & & & 0 \\
\hline \multirow{8}{*}{$90^{\circ}$} & {$\left[0^{\circ}, 45^{\circ}\right]$} & & & 1 \\
\hline & {$\left[45^{\circ}, 90^{\circ}\right]$} & & & 1 \\
\hline & {$\left[90^{\circ}, 135^{\circ}\right]$} & & & 1 \\
\hline & {$\left[135^{\circ}, 180^{\circ}\right]$} & & & 1 \\
\hline & {$\left[180^{\circ}, 225^{\circ}\right]$} & & & 1 \\
\hline & {$\left[225^{\circ}, 270^{\circ}\right]$} & & & 1 \\
\hline & {$\left[270^{\circ}, 315^{\circ}\right]$} & & & 1 \\
\hline & {$\left[315^{\circ}, 360^{\circ}\right]$} & & & 1 \\
\hline \multirow{8}{*}{$-90^{\circ}$} & {$\left[0^{\circ}, 45^{\circ}\right]$} & & & 1 \\
\hline & {$\left[45^{\circ}, 90^{\circ}\right]$} & & & 1 \\
\hline & {$\left[90^{\circ}, 135^{\circ}\right]$} & & & 1 \\
\hline & {$\left[135^{\circ}, 180^{\circ}\right]$} & & & 1 \\
\hline & {$\left[180^{\circ}, 225^{\circ}\right]$} & & & 1 \\
\hline & {$\left[225^{\circ}, 270^{\circ}\right]$} & & & 1 \\
\hline & {$\left[270^{\circ}, 315^{\circ}\right]$} & & & 1 \\
\hline & {$\left[315^{\circ}, 360^{\circ}\right]$} & & & 1 \\
\hline
\end{tabular}


Table 2 Average errors of various blended high-resolution schemes at different Courant numbers for Test 1

\begin{tabular}{cccccccc}
\hline Scheme & $\mathrm{Co}=0.2$ & $\mathrm{Co}=0.3$ & $\mathrm{Co}=0.4$ & $\mathrm{Co}=0.5$ & $\mathrm{Co}=0.6$ & $\mathrm{Co}=0.7$ & $\mathrm{Co}=0.8$ \\
\hline CICSAM & $1.047 \mathrm{E}-03$ & $1.567 \mathrm{E}-03$ & $2.501 \mathrm{E}-03$ & $4.778 \mathrm{E}-03$ & $1.047 \mathrm{E}-02$ & $2.049 \mathrm{E}-02$ & $2.956 \mathrm{E}-02$ \\
HRIC & $5.343 \mathrm{E}-03$ & $5.149 \mathrm{E}-03$ & $9.118 \mathrm{E}-03$ & $1.900 \mathrm{E}-02$ & $3.087 \mathrm{E}-02$ & $3.690 \mathrm{E}-02$ & $3.994 \mathrm{E}-02$ \\
THOR & $1.918 \mathrm{E}-03$ & $2.997 \mathrm{E}-03$ & $4.811 \mathrm{E}-03$ & $9.259 \mathrm{E}-03$ & $1.830 \mathrm{E}-02$ & $2.941 \mathrm{E}-02$ & $3.785 \mathrm{E}-02$ \\
STACS & $5.344 \mathrm{E}-03$ & $5.414 \mathrm{E}-03$ & $5.461 \mathrm{E}-03$ & $5.546 \mathrm{E}-03$ & $5.855 \mathrm{E}-03$ & $6.145 \mathrm{E}-03$ & $6.456 \mathrm{E}-03$ \\
M-CICSAM & $1.011 \mathrm{E}-03$ & $1.421 \mathrm{E}-03$ & $2.312 \mathrm{E}-03$ & $2.452 \mathrm{E}-03$ & $2.541 \mathrm{E}-03$ & $4.750 \mathrm{E}-03$ & $5.037 \mathrm{E}-03$ \\
\hline
\end{tabular}

Table 3 Average errors of various blended high-resolution schemes at different Courant numbers for Test 2

\begin{tabular}{cccccccc}
\hline Scheme & $\mathrm{Co}=0.2$ & $\mathrm{Co}=0.3$ & $\mathrm{Co}=0.4$ & $\mathrm{Co}=0.5$ & $\mathrm{Co}=0.6$ & $\mathrm{Co}=0.7$ & $\mathrm{Co}=0.8$ \\
\hline CICSAM & $3.406 \mathrm{E}-03$ & $3.858 \mathrm{E}-03$ & $5.295 \mathrm{E}-03$ & $1.056 \mathrm{E}-02$ & $1.956 \mathrm{E}-02$ & $2.553 \mathrm{E}-02$ & $2.918 \mathrm{E}-02$ \\
HRIC & $5.122 \mathrm{E}-03$ & $6.208 \mathrm{E}-03$ & $1.519 \mathrm{E}-02$ & $2.490 \mathrm{E}-02$ & $2.997 \mathrm{E}-02$ & $3.142 \mathrm{E}-02$ & $3.170 \mathrm{E}-02$ \\
THOR & $3.967 \mathrm{E}-03$ & $6.376 \mathrm{E}-03$ & $1.189 \mathrm{E}-02$ & $1.747 \mathrm{E}-02$ & $2.575 \mathrm{E}-02$ & $3.270 \mathrm{E}-02$ & $3.708 \mathrm{E}-02$ \\
STACS & $6.038 \mathrm{E}-03$ & $6.184 \mathrm{E}-03$ & $6.366 \mathrm{E}-03$ & $6.554 \mathrm{E}-03$ & $6.727 \mathrm{E}-03$ & $6.980 \mathrm{E}-03$ & $7.138 \mathrm{E}-03$ \\
M-CICSAM & $3.353 \mathrm{E}-03$ & $3.471 \mathrm{E}-03$ & $4.236 \mathrm{E}-03$ & $4.651 \mathrm{E}-03$ & $4.662 \mathrm{E}-03$ & $5.556 \mathrm{E}-03$ & $5.725 \mathrm{E}-03$ \\
\hline
\end{tabular}

Table 4 Average errors of various blended high-resolution schemes at different Courant numbers for Test 3

\begin{tabular}{cccccccc}
\hline Scheme & $\mathrm{Co}=0.2$ & $\mathrm{Co}=0.3$ & $\mathrm{Co}=0.4$ & $\mathrm{Co}=0.5$ & $\mathrm{Co}=0.6$ & $\mathrm{Co}=0.7$ & $\mathrm{Co}=0.8$ \\
\hline CICSAM & $2.014 \mathrm{E}-02$ & $2.162 \mathrm{E}-02$ & $2.677 \mathrm{E}-02$ & $3.348 \mathrm{E}-02$ & $4.375 \mathrm{E}-02$ & $5.528 \mathrm{E}-02$ & $6.607 \mathrm{E}-02$ \\
HRIC & $2.662 \mathrm{E}-02$ & $2.948 \mathrm{E}-02$ & $4.199 \mathrm{E}-02$ & $5.592 \mathrm{E}-02$ & $7.041 \mathrm{E}-02$ & $8.110 \mathrm{E}-02$ & $8.757 \mathrm{E}-02$ \\
THOR & $2.060 \mathrm{E}-02$ & $2.227 \mathrm{E}-02$ & $2.780 \mathrm{E}-02$ & $3.904 \mathrm{E}-02$ & $5.439 \mathrm{E}-02$ & $6.821 \mathrm{E}-02$ & $7.986 \mathrm{E}-02$ \\
STACS & $3.383 \mathrm{E}-02$ & $3.493 \mathrm{E}-02$ & $3.496 \mathrm{E}-02$ & $3.516 \mathrm{E}-02$ & $3.545 \mathrm{E}-02$ & $3.556 \mathrm{E}-02$ & $3.563 \mathrm{E}-02$ \\
M-CICSAM & $1.379 \mathrm{E}-02$ & $1.464 \mathrm{E}-02$ & $1.552 \mathrm{E}-02$ & $1.708 \mathrm{E}-02$ & $1.949 \mathrm{E}-02$ & $2.448 \mathrm{E}-02$ & $2.687 \mathrm{E}-02$ \\
\hline
\end{tabular}

Table 5 Average errors of various blended high-resolution schemes at different Courant numbers for Test 4

\begin{tabular}{cccccccc}
\hline Scheme & $\mathrm{Co}=0.2$ & $\mathrm{Co}=0.3$ & $\mathrm{Co}=0.4$ & $\mathrm{Co}=0.5$ & $\mathrm{Co}=0.6$ & $\mathrm{Co}=0.7$ & $\mathrm{Co}=0.8$ \\
\hline CICSAM & $6.642 \mathrm{E}-03$ & $1.088 \mathrm{E}-02$ & $1.766 \mathrm{E}-02$ & $3.877 \mathrm{E}-02$ & $7.123 \mathrm{E}-02$ & $9.927 \mathrm{E}-02$ & $1.222 \mathrm{E}-01$ \\
HRIC & $7.320 \mathrm{E}-03$ & $1.348 \mathrm{E}-02$ & $4.170 \mathrm{E}-02$ & $7.771 \mathrm{E}-02$ & $1.060 \mathrm{E}-01$ & $1.275 \mathrm{E}-01$ & $1.442 \mathrm{E}-01$ \\
THOR & $5.173 \mathrm{E}-03$ & $6.812 \mathrm{E}-03$ & $1.439 \mathrm{E}-02$ & $3.804 \mathrm{E}-02$ & $7.493 \mathrm{E}-02$ & $1.061 \mathrm{E}-01$ & $1.304 \mathrm{E}-01$ \\
STACS & $1.213 \mathrm{E}-02$ & $1.542 \mathrm{E}-02$ & $1.899 \mathrm{E}-02$ & $2.365 \mathrm{E}-02$ & $2.981 \mathrm{E}-02$ & $3.733 \mathrm{E}-02$ & $3.990 \mathrm{E}-02$ \\
M-CICSAM & $3.973 \mathrm{E}-03$ & $5.373 \mathrm{E}-03$ & $6.144 \mathrm{E}-03$ & $8.588 \mathrm{E}-03$ & $1.381 \mathrm{E}-02$ & $2.294 \mathrm{E}-02$ & $2.707 \mathrm{E}-02$ \\
\hline
\end{tabular}




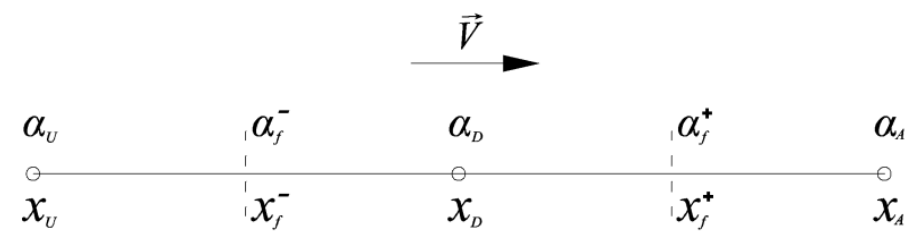

Figure 1. Schematic representation of the NVD/NVSF nomenclature.

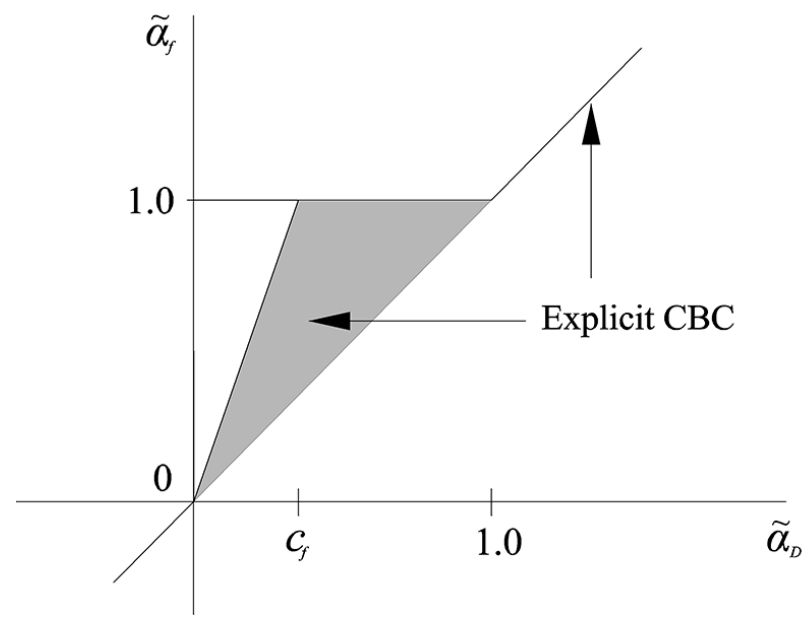

Figure 2. The Explicit CBC in the normalized variable diagram (NVD).
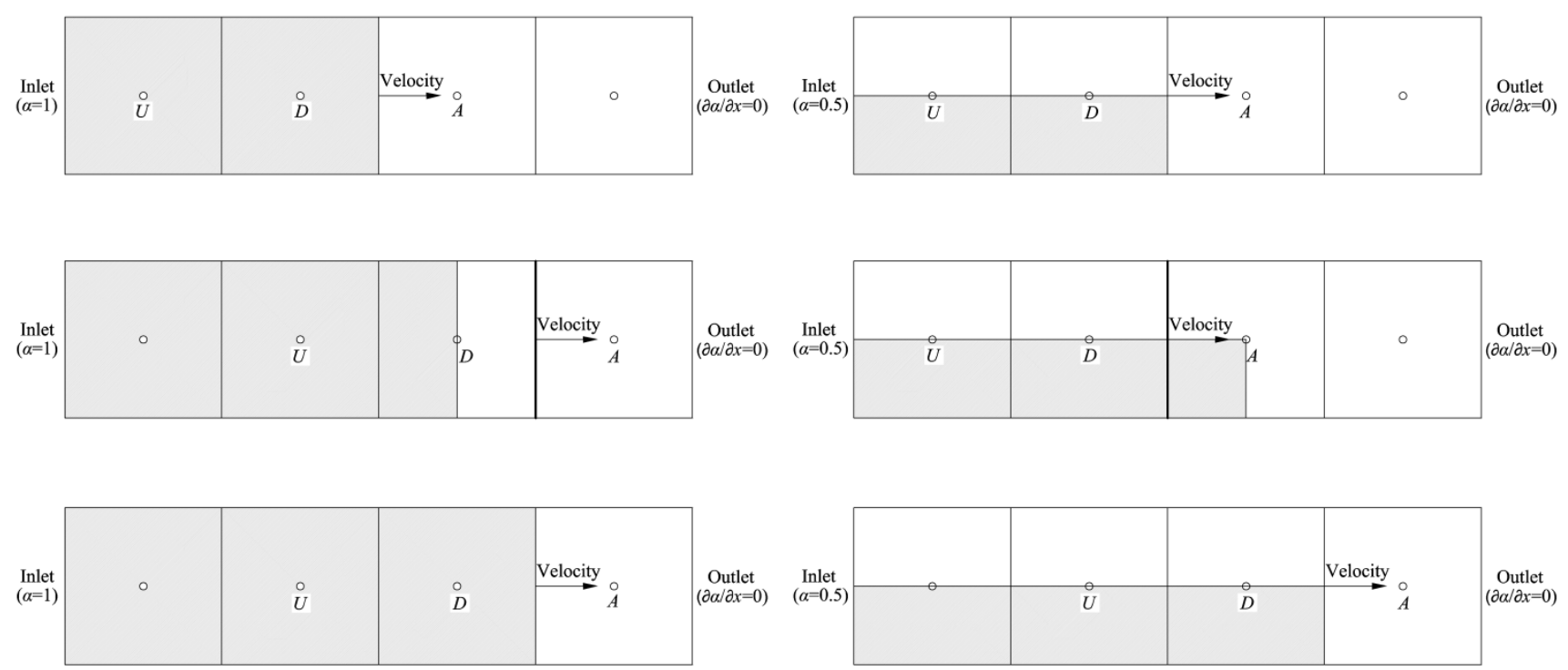

(a)

(b)

Figure 3. Advection of a fluid block at a Courant number of 0.5 using the original switching weighting function described by Eq. (22) for one-dimensional flow:

(a) the interface is parallel to the cell face; (b) the interface is perpendicular to the cell face. 


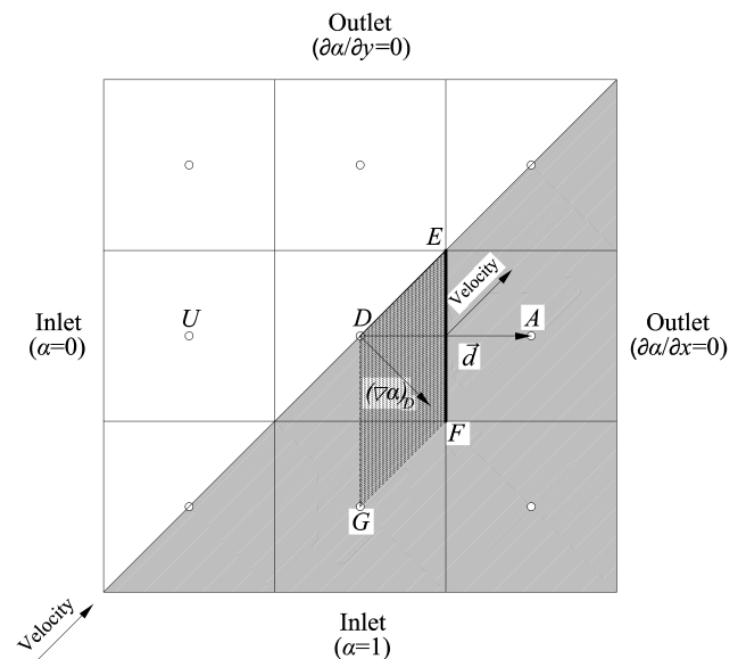

Figure 4. Advection of an interface at a Courant number of 0.5 in an oblique velocity field for two-dimensional flow.
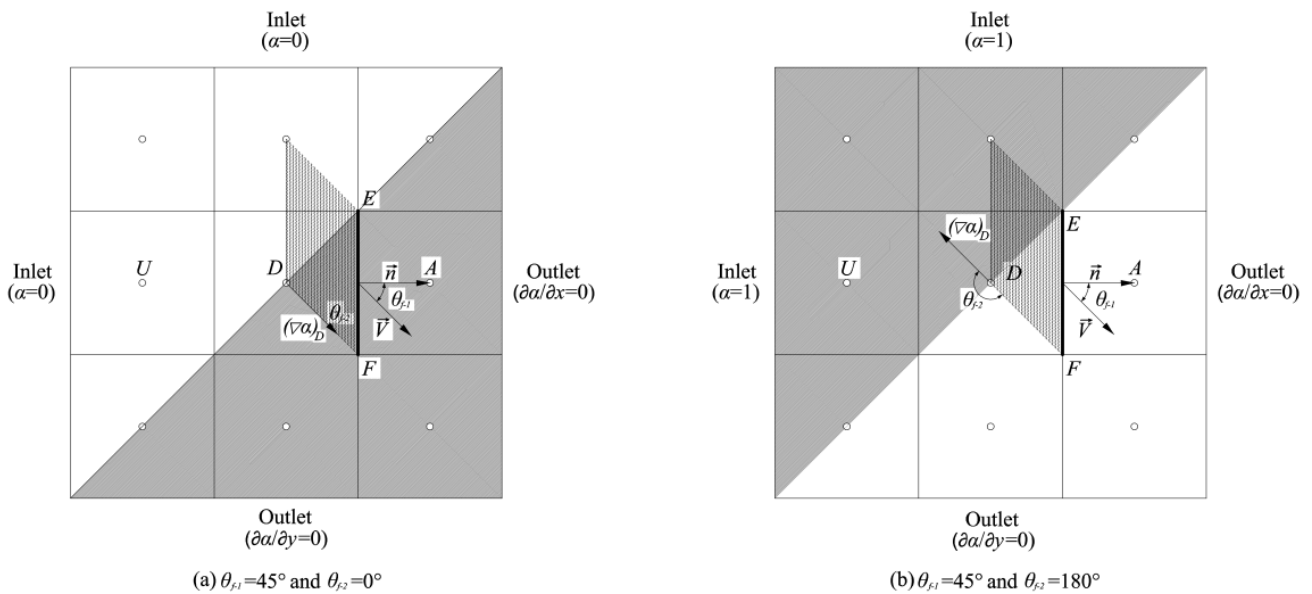

Figure 5. The exact volumetric fluxes of volume fraction advected through the highlighted cell face $E F$ for $\theta_{f-1}=45^{\circ}$ : (a) $\theta_{f-2}=0^{\circ}$; (b) $\theta_{f-2}=180^{\circ}$.

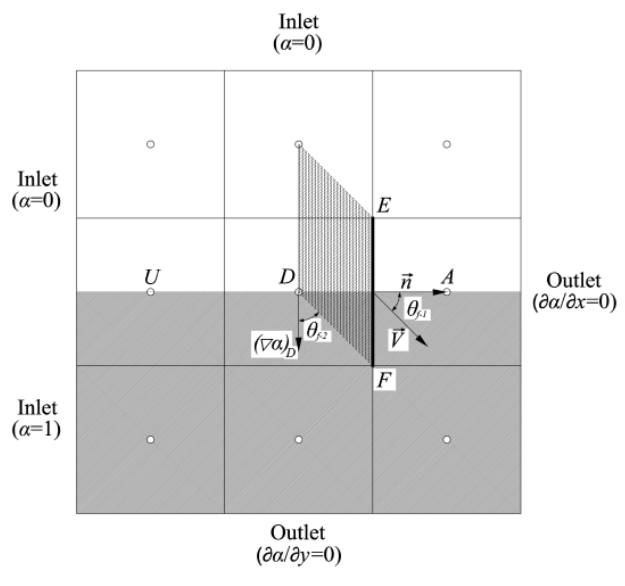

(a) $\theta_{f, t}=45^{\circ}$ and $\theta_{f 3}=45^{\circ}$

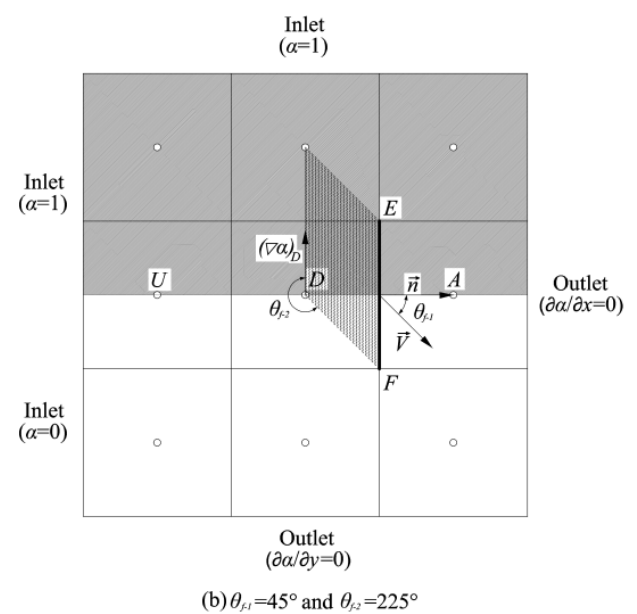

(b) $\theta_{f 1}=45^{\circ}$ and $\theta_{k 2}=225^{\circ}$

Figure 6. The exact volumetric fluxes of volume fraction advected through the highlighted cell face $E F$ for $\theta_{f-1}=45^{\circ}$ : (a) $\theta_{f-2}=45^{\circ}$; (b) $\theta_{f-2}=225^{\circ}$. 


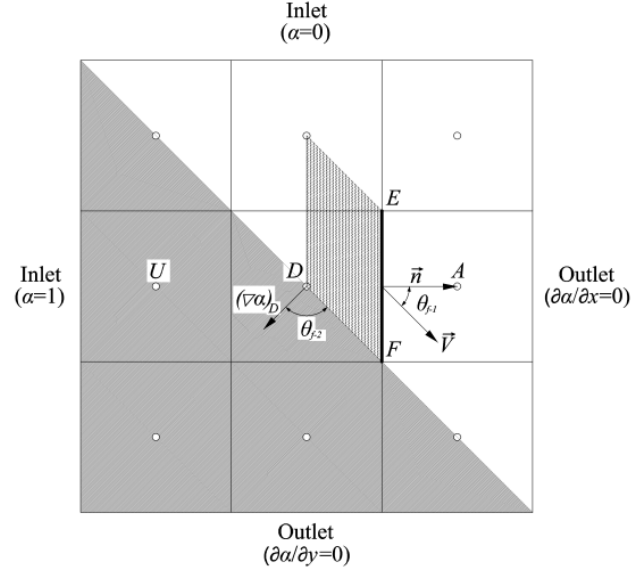

(a) $\theta_{f l}=45^{\circ}$ and $\theta_{f 2}=90^{\circ}$

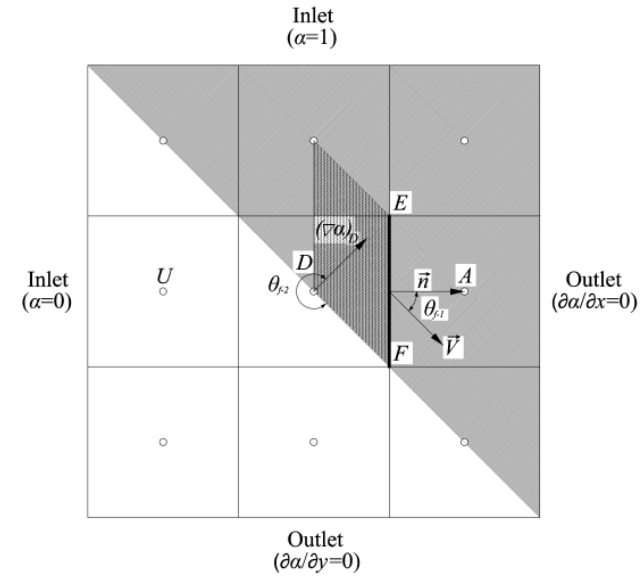

(b) $\theta_{f t}=45^{\circ}$ and $\theta_{f 2}=270^{\circ}$

Figure 7. The exact volumetric fluxes of volume fraction advected through the highlighted cell face $E F$ for $\theta_{f-1}=45^{\circ}$ : (a) $\theta_{f-2}=90^{\circ}$; (b) $\theta_{f-2}=270^{\circ}$.

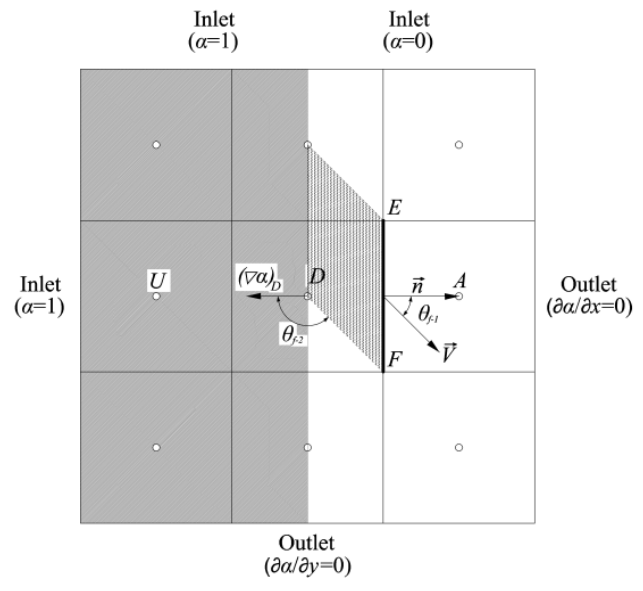

(a) $\theta_{f 1}=45^{\circ}$ and $\theta_{f 2}=135^{\circ}$

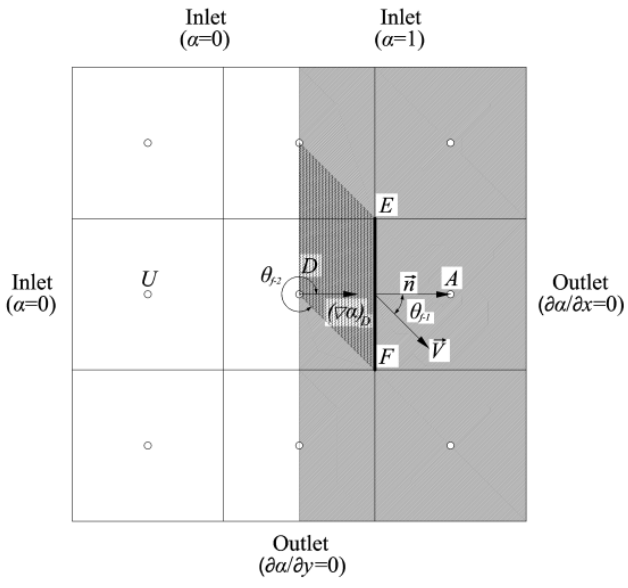

(b) $\theta_{f 1}=45^{\circ}$ and $\theta_{f 2}=315^{\circ}$

Figure 8. The exact volumetric fluxes of volume fraction advected through the highlighted cell face $E F$ for $\theta_{f-1}=45^{\circ}$ : (a) $\theta_{f-2}=135^{\circ}$; (b) $\theta_{f-2}=315^{\circ}$.

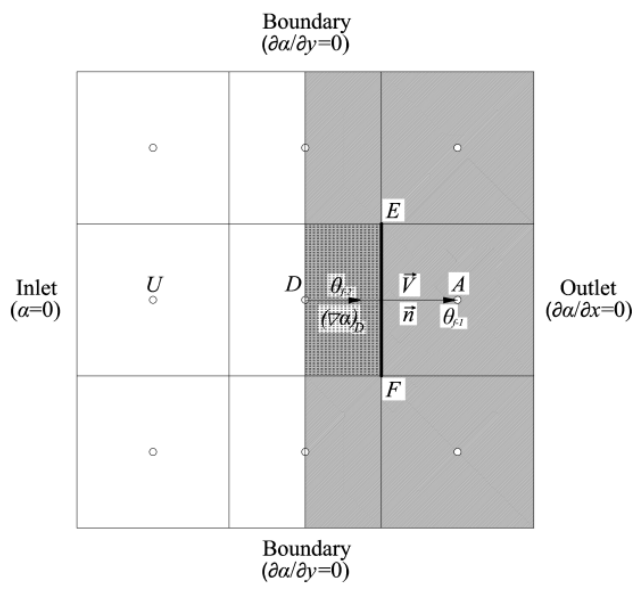

(a) $\theta_{f L}=0^{\circ}$ and $\theta_{f 2}=0^{\circ}$

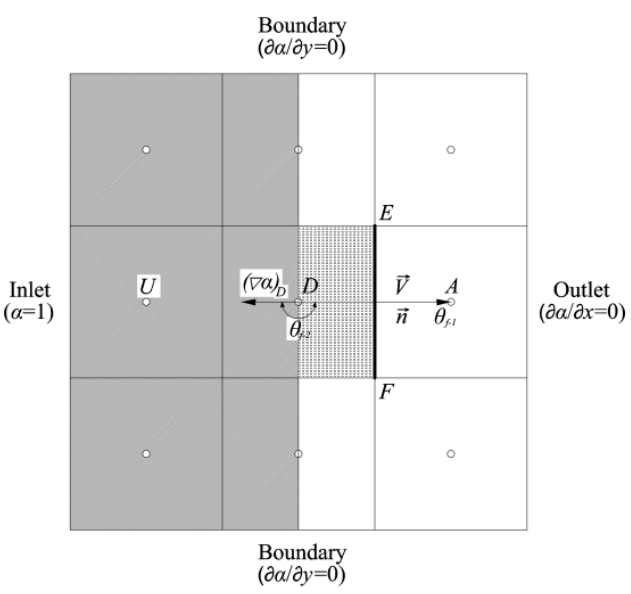

(b) $\theta_{f t}=0^{\circ}$ and $\theta_{f 2}=180^{\circ}$

Figure 9. The exact volumetric fluxes of volume fraction advected through the highlighted cell face $E F$ for $\theta_{f-1}=0^{\circ}$ : (a) $\theta_{f-2}=0^{\circ}$; (b) $\theta_{f-2}=180^{\circ}$. 

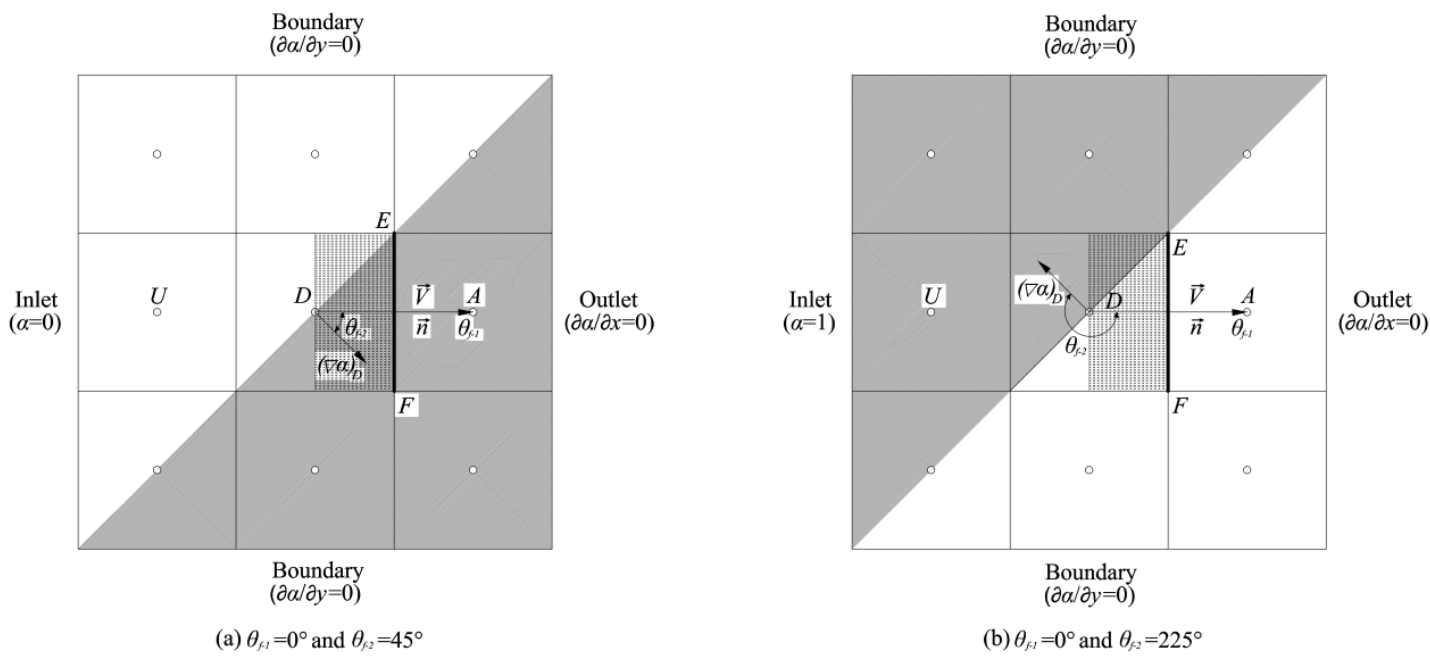

Figure 10. The exact volumetric fluxes of volume fraction advected through the highlighted cell face $E F$ for $\theta_{f-1}=0^{\circ}$ : (a) $\theta_{f-2}=45^{\circ}$; (b) $\theta_{f-2}=225^{\circ}$.

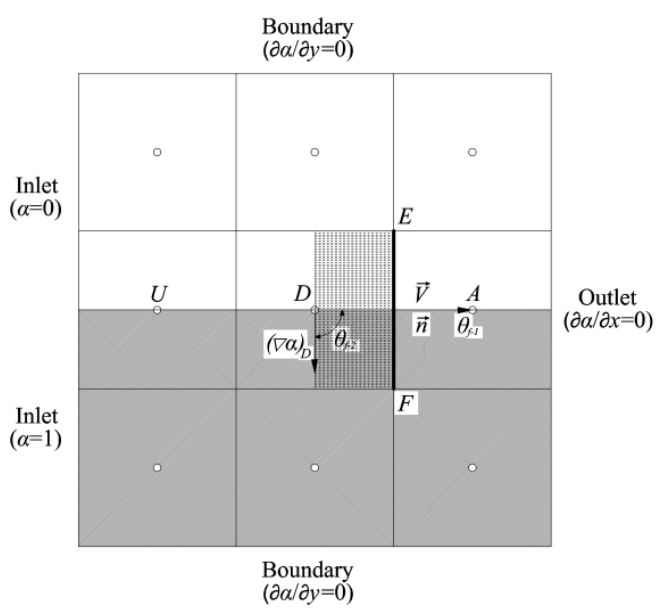

(a) $\theta_{f r}=0^{\circ}$ and $\theta_{f 2}=90^{\circ}$

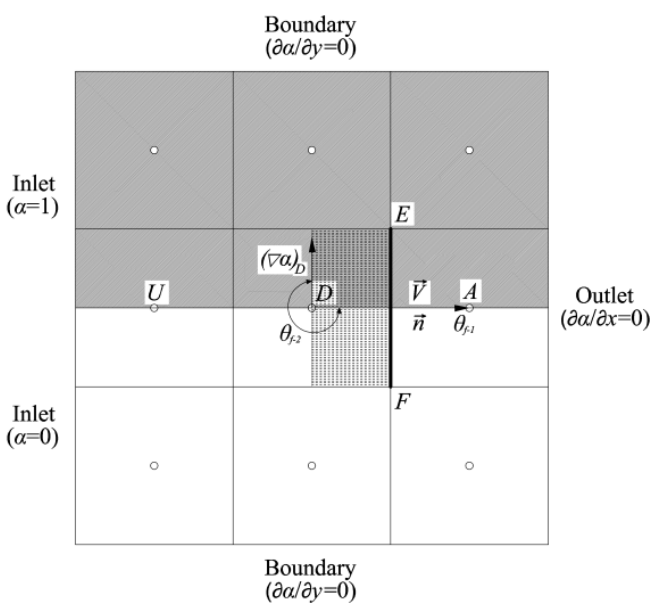

(b) $\theta_{f 1}=0^{\circ}$ and $\theta_{f 2}=270^{\circ}$

Figure 11. The exact volumetric fluxes of volume fraction advected through the highlighted cell face $E F$ for $\theta_{f-1}=0^{\circ}$ : (a) $\theta_{f-2}=90^{\circ}$; (b) $\theta_{f-2}=270^{\circ}$.

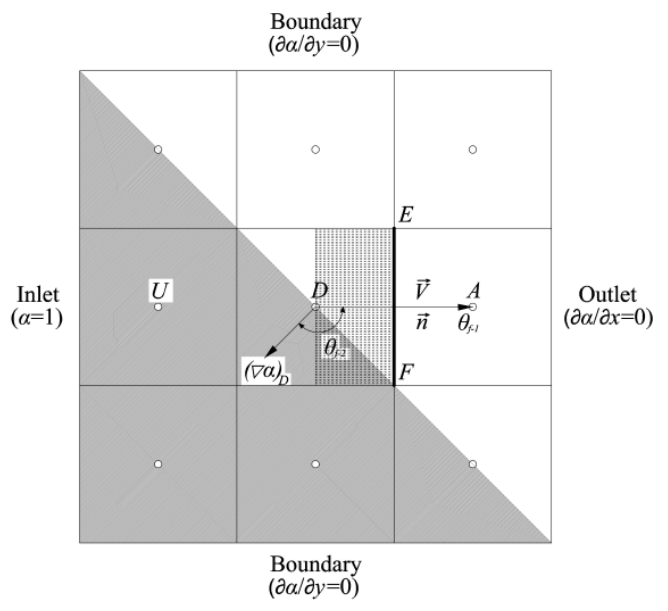

(a) $\theta_{f, 1}=0^{\circ}$ and $\theta_{f: 2}=135^{\circ}$

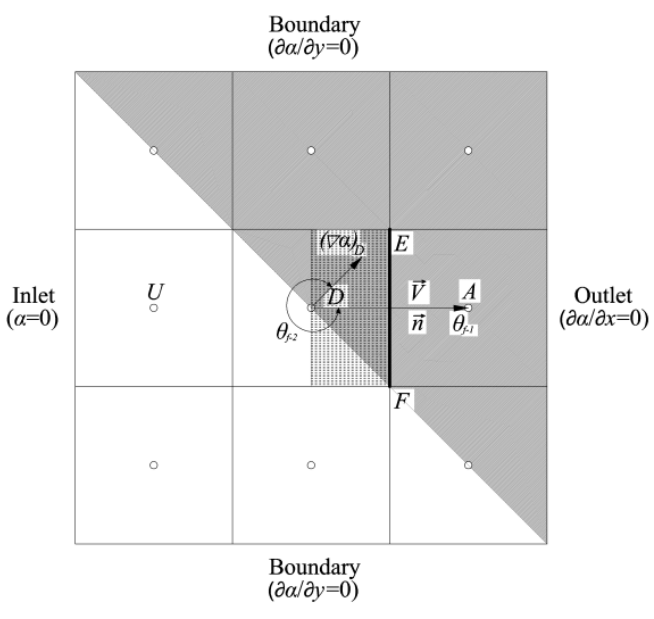

(b) $\theta_{f, 1}=0^{\circ}$ and $\theta_{f, 2}=315^{\circ}$

Figure 12. The exact volumetric fluxes of volume fraction advected through the highlighted cell face $E F$ for $\theta_{f-1}=0^{\circ}$ : (a) $\theta_{f-2}=135^{\circ}$; (b) $\theta_{f-2}=315^{\circ}$. 


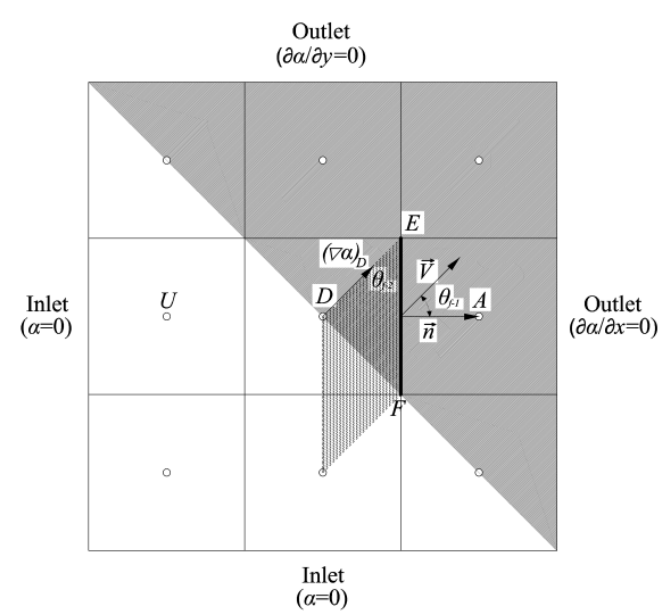

(a) $\theta_{f l}=-45^{\circ}$ and $\theta_{f 2}=0^{\circ}$

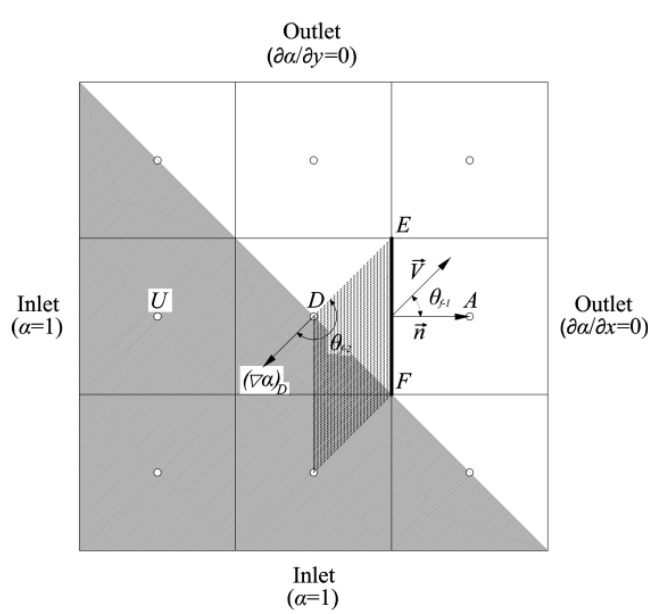

(a) $\theta_{f t}=-45^{\circ}$ and $\theta_{f^{2}}=180^{\circ}$

Figure 13. The exact volumetric fluxes of volume fraction advected through the highlighted cell face $E F$ for $\theta_{f-1}=-45^{\circ}$ : (a) $\theta_{f-2}=0^{\circ}$; (b) $\theta_{f-2}=180^{\circ}$.
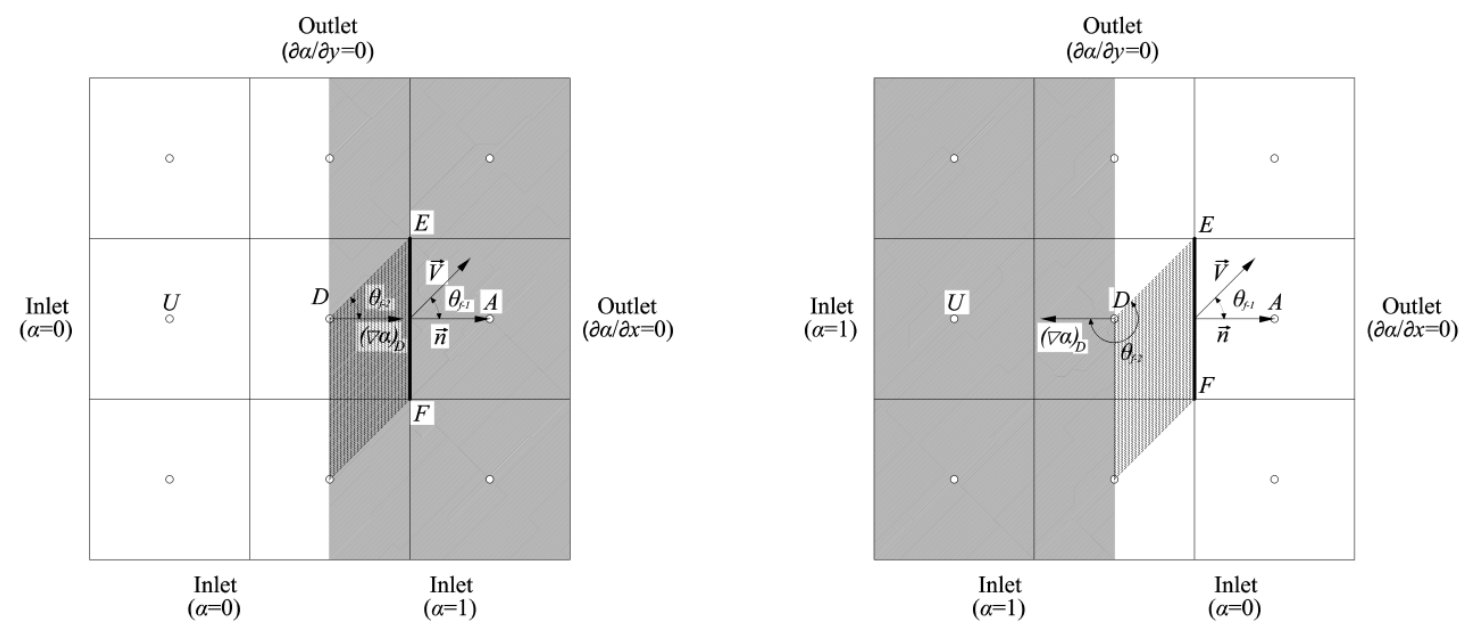

(a) $\theta_{f i}=-45^{\circ}$ and $\theta_{f 2}=45^{\circ}$

(a) $\theta_{k 1}=-45^{\circ}$ and $\theta_{\beta 2}=225^{\circ}$

Figure 14. The exact volumetric fluxes of volume fraction advected through the highlighted cell face $E F$ for $\theta_{f-1}=-45^{\circ}$ : (a) $\theta_{f-2}=45^{\circ}$; (b) $\theta_{f-2}=225^{\circ}$.

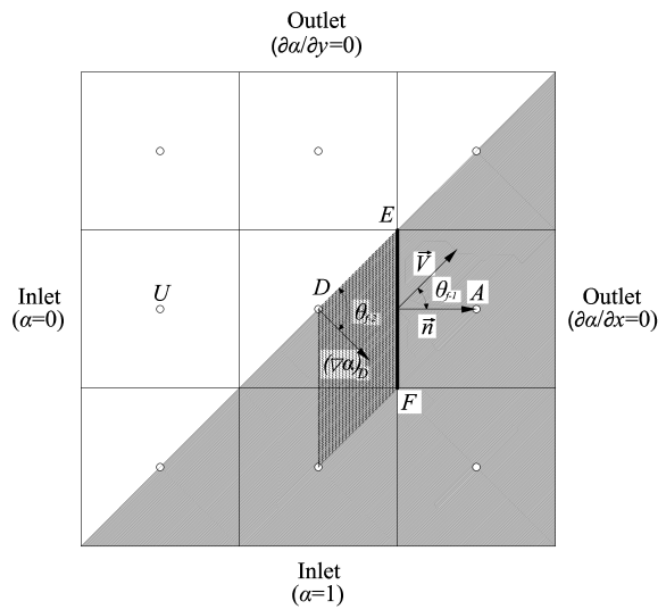

(a) $\theta_{f 1}=-45^{\circ}$ and $\theta_{f 2}=90^{\circ}$

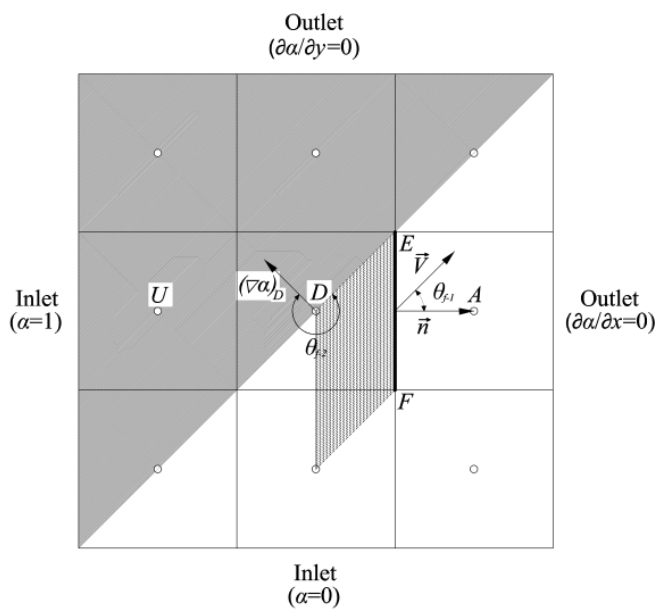

(a) $\theta_{f 1}=-45^{\circ}$ and $\theta_{r 2}=270^{\circ}$

Figure 15. The exact volumetric fluxes of volume fraction advected through the highlighted cell face $E F$ for $\theta_{f-1}=-45^{\circ}$ : (a) $\theta_{f-2}=90^{\circ}$; (b) $\theta_{f-2}=270^{\circ}$. 


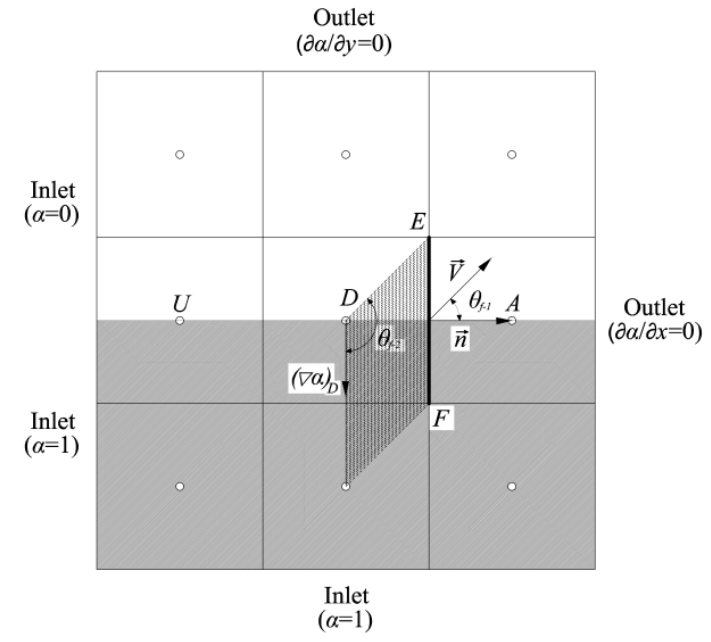

(a) $\theta_{f-1}=-45^{\circ}$ and $\theta_{f 2}=135^{\circ}$

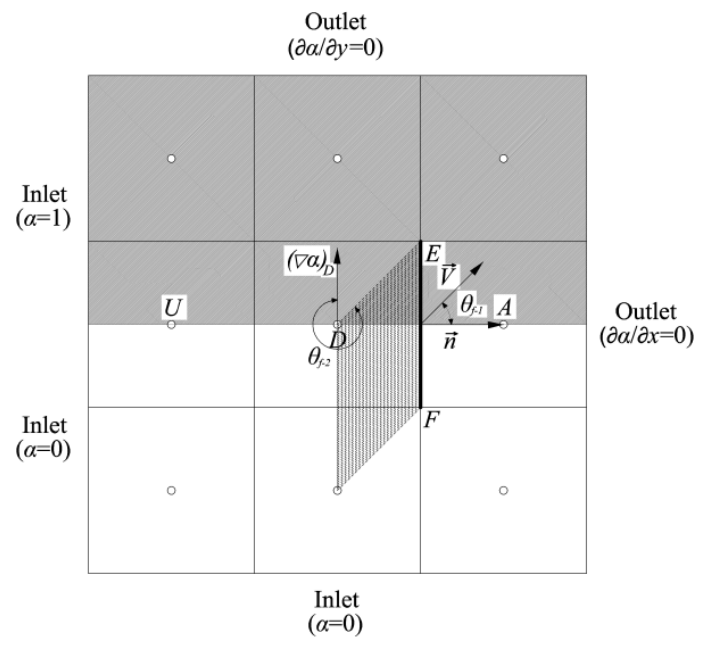

(a) $\theta_{f-1}=-45^{\circ}$ and $\theta_{f 2}=315^{\circ}$

Figure 16. The exact volumetric fluxes of volume fraction advected through the highlighted cell face $E F$ for $\theta_{f-1}=-45^{\circ}$ : (a) $\theta_{f-2}=135^{\circ}$; (b) $\theta_{f-2}=315^{\circ}$.

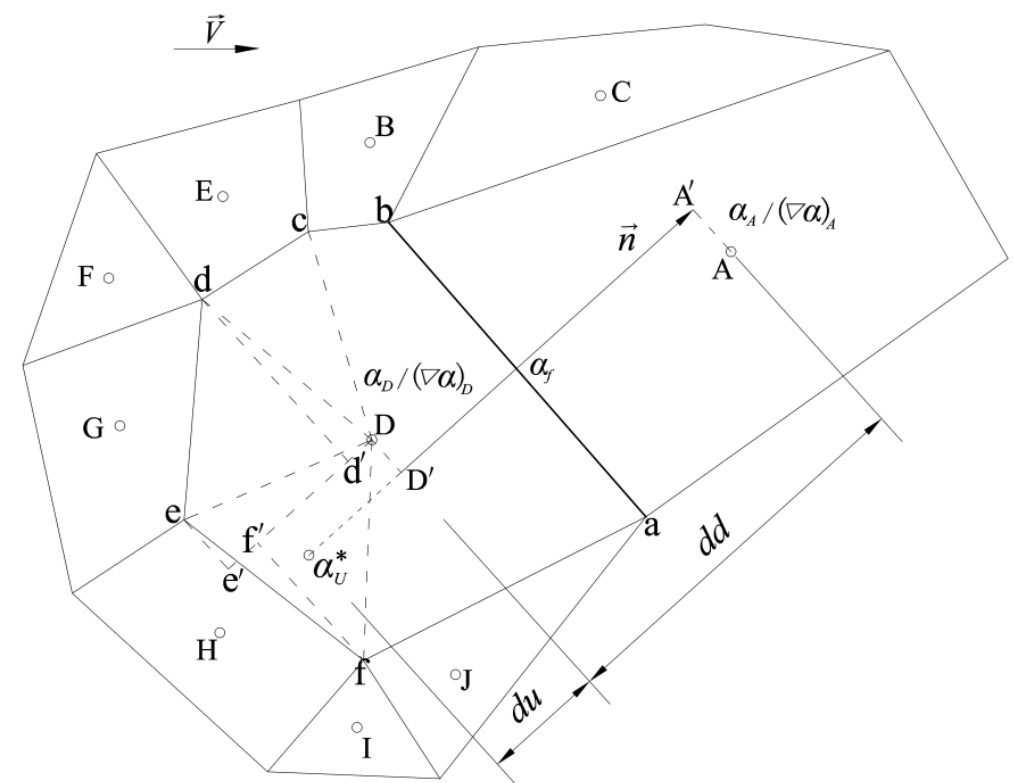

Figure 17. The predictor step of the New-Technique-2 reconstruction technique.

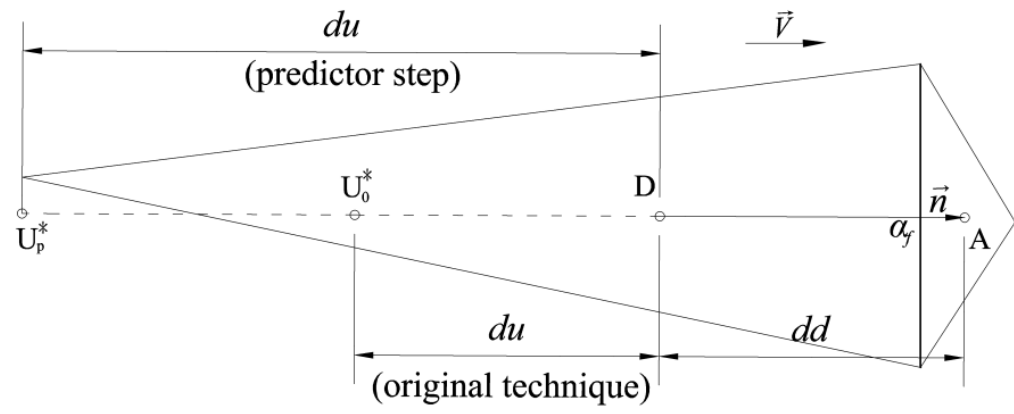

Figure 18. The corrector step of the New-Technique-2 reconstruction technique. 


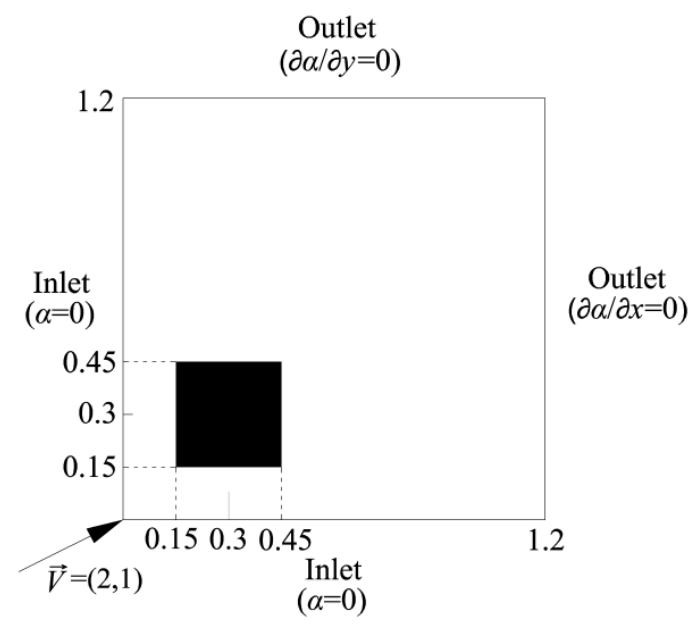

Figure 19. Schematic representation of the advected square droplet.

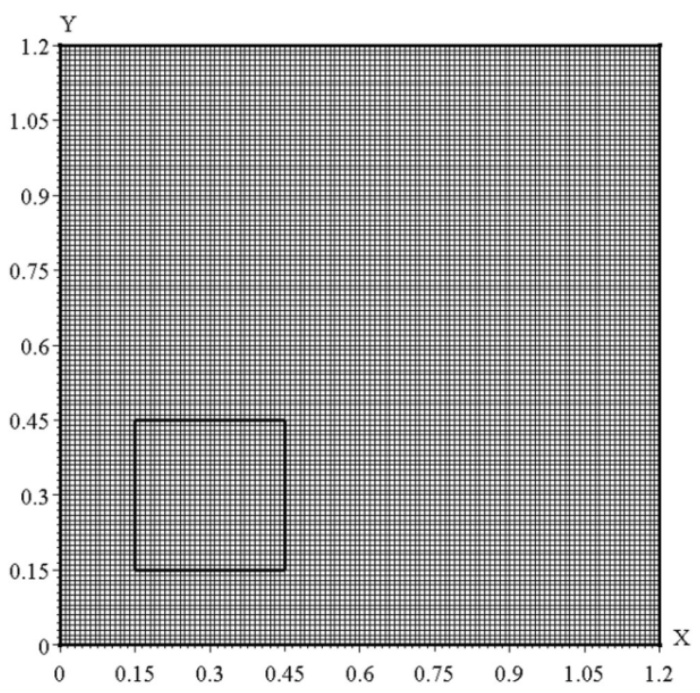

Figure 20. Physical domain and structured mesh used for the advection of a square droplet in an oblique uniform velocity field.

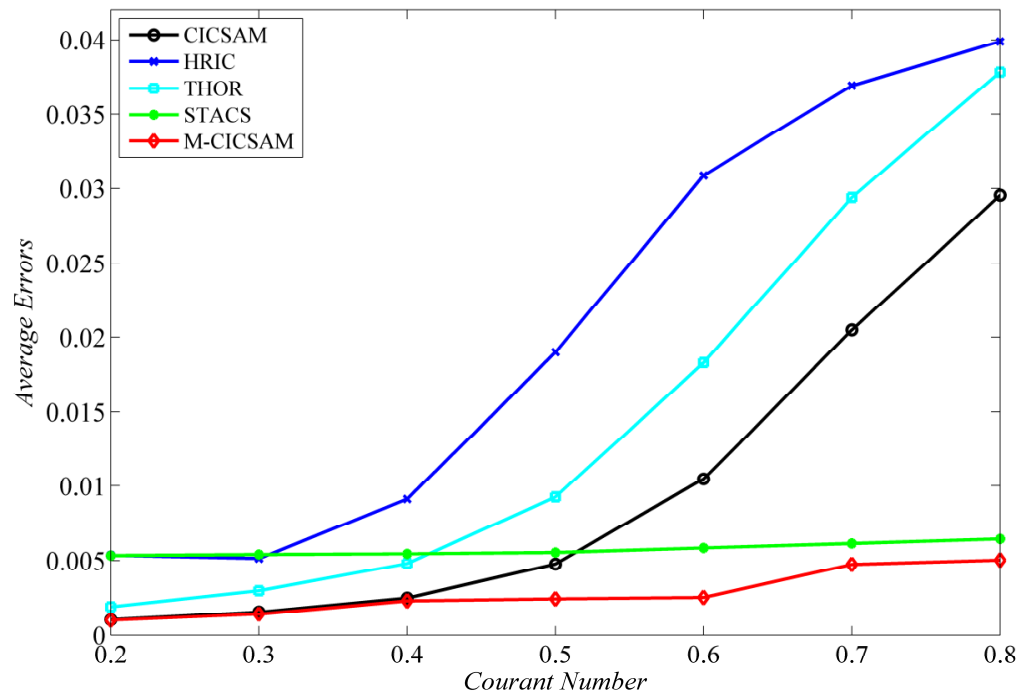

Figure 21. Comparison of accuracy of various blended high-resolution schemes at different Courant numbers in Test 1. 


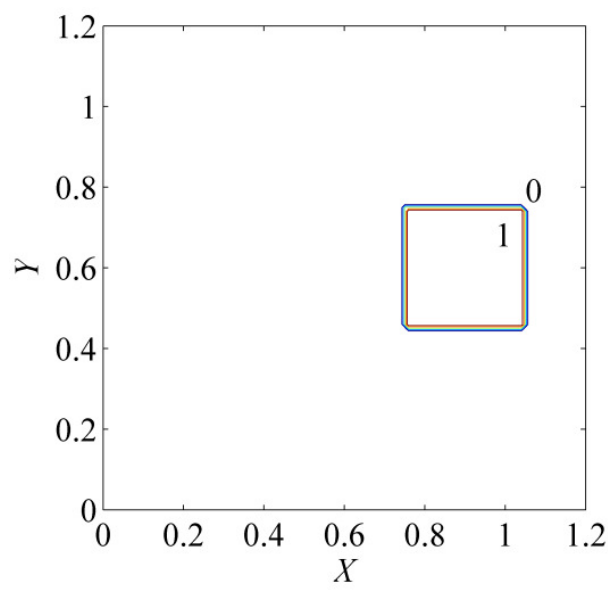

(a) The Exact Solution

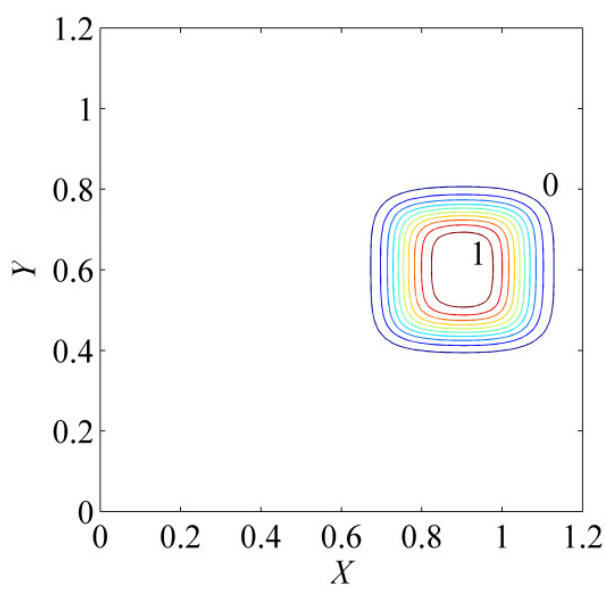

(b) HRIC

Figure 22. Contour plots of the advected square droplet at a Courant number of 0.6: (a) the exact solution; (b) HRIC.

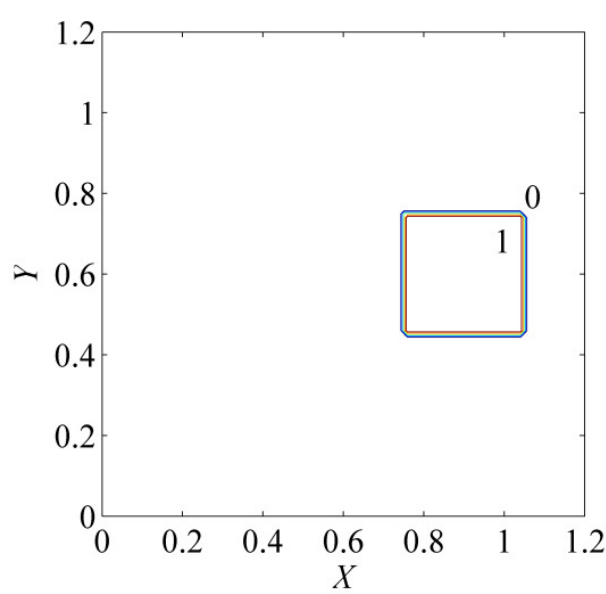

(a) The Exact Solution

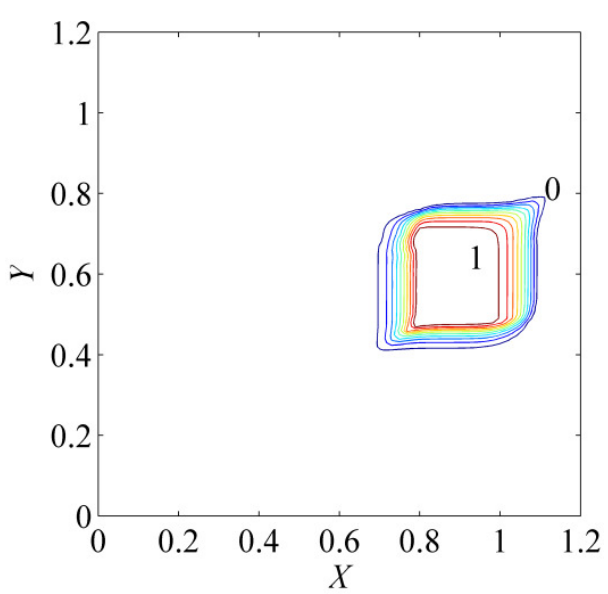

(b) THOR

Figure 23. Contour plots of the advected square droplet at a Courant number of 0.6:

(a) the exact solution; (b) THOR.

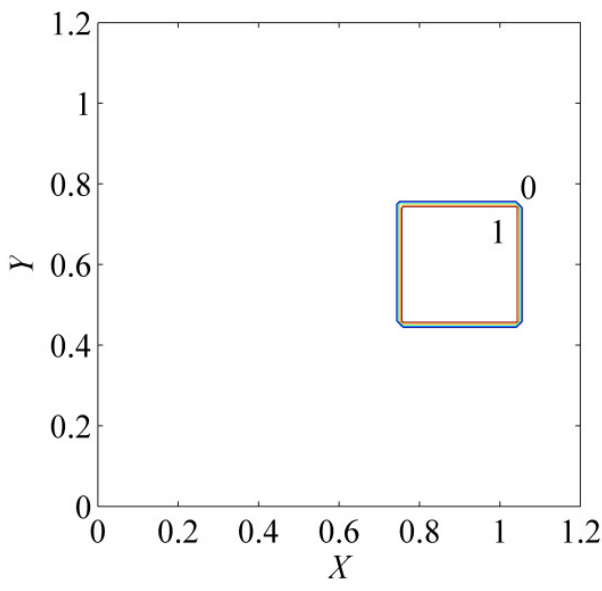

(a) The Exact Solution

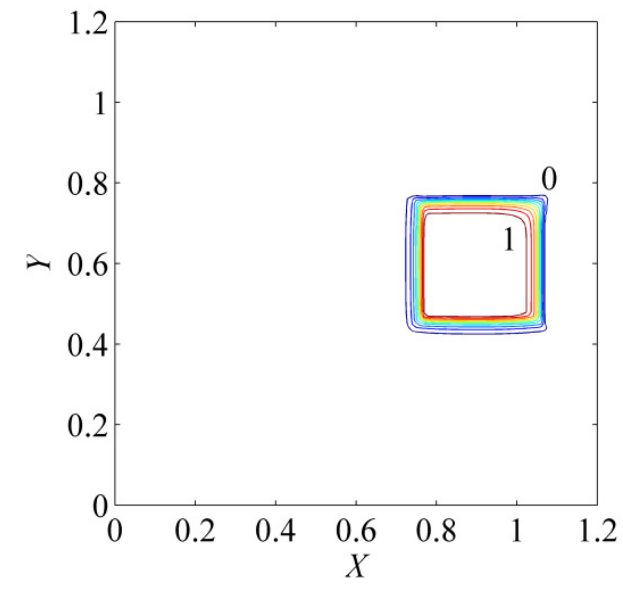

(b) CICSAM

Figure 24. Contour plots of the advected square droplet at a Courant number of 0.6:

(a) the exact solution; (b) CICSAM. 


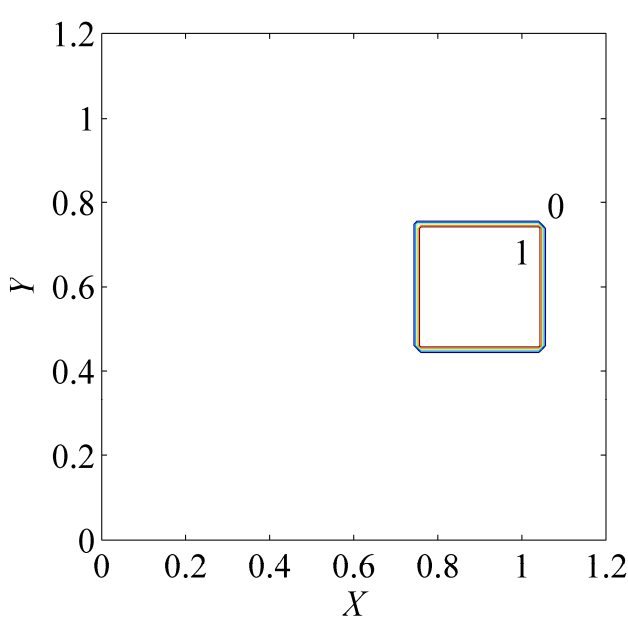

(a) The Exact Solution

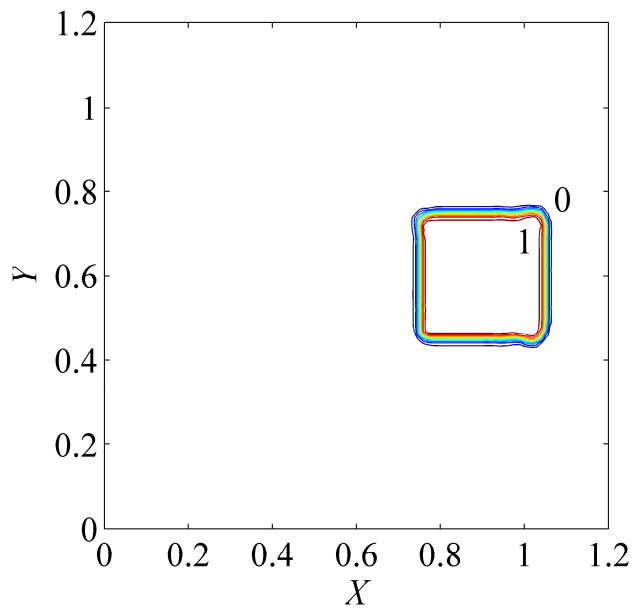

(b) STACS

Figure 25. Contour plots of the advected square droplet at a Courant number of 0.6: (a) the exact solution; (b) STACS.

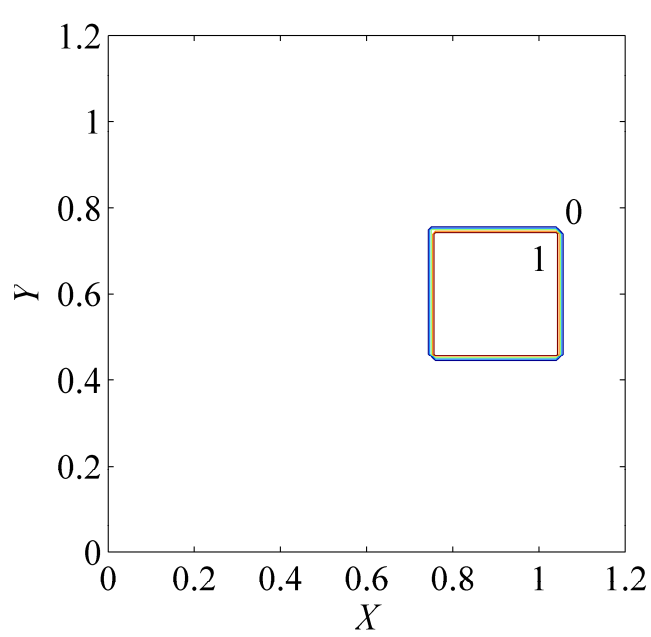

(a) The Exact Solution

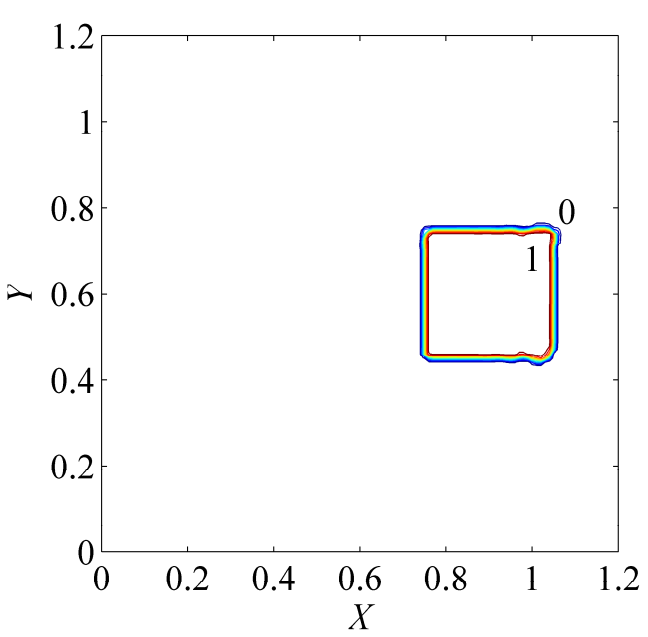

(b) M-CICSAM

Figure 26. Contour plots of the advected square droplet at a Courant number of 0.6:

(a) the exact solution; (b) M-CICSAM.

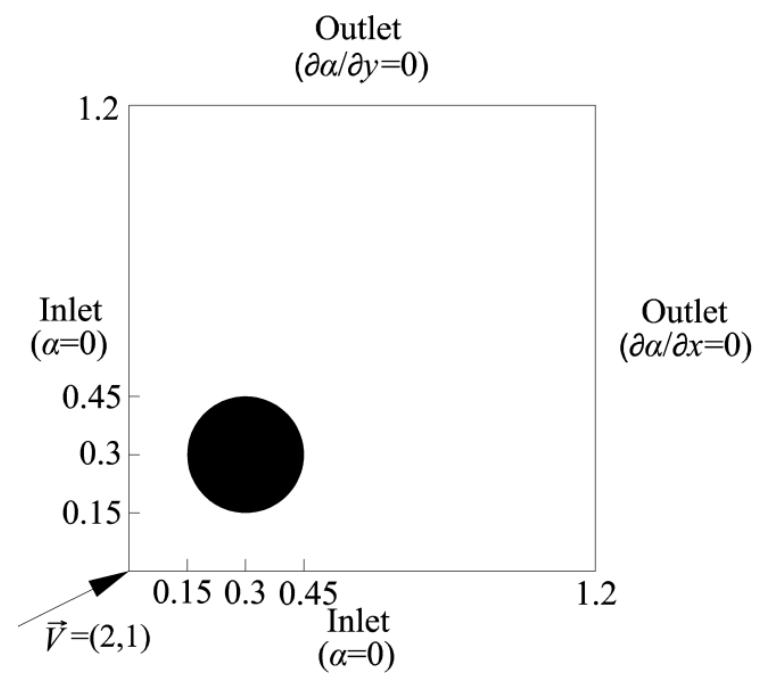

Figure 27. Schematic representation of the advected round droplet. 


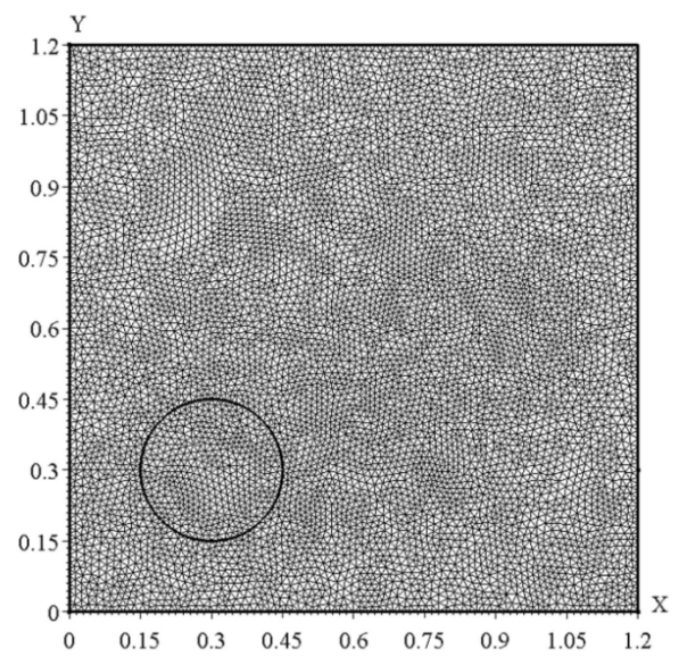

Figure 28. Physical domain and unstructured mesh used for the advection of a round droplet in an oblique uniform velocity field.

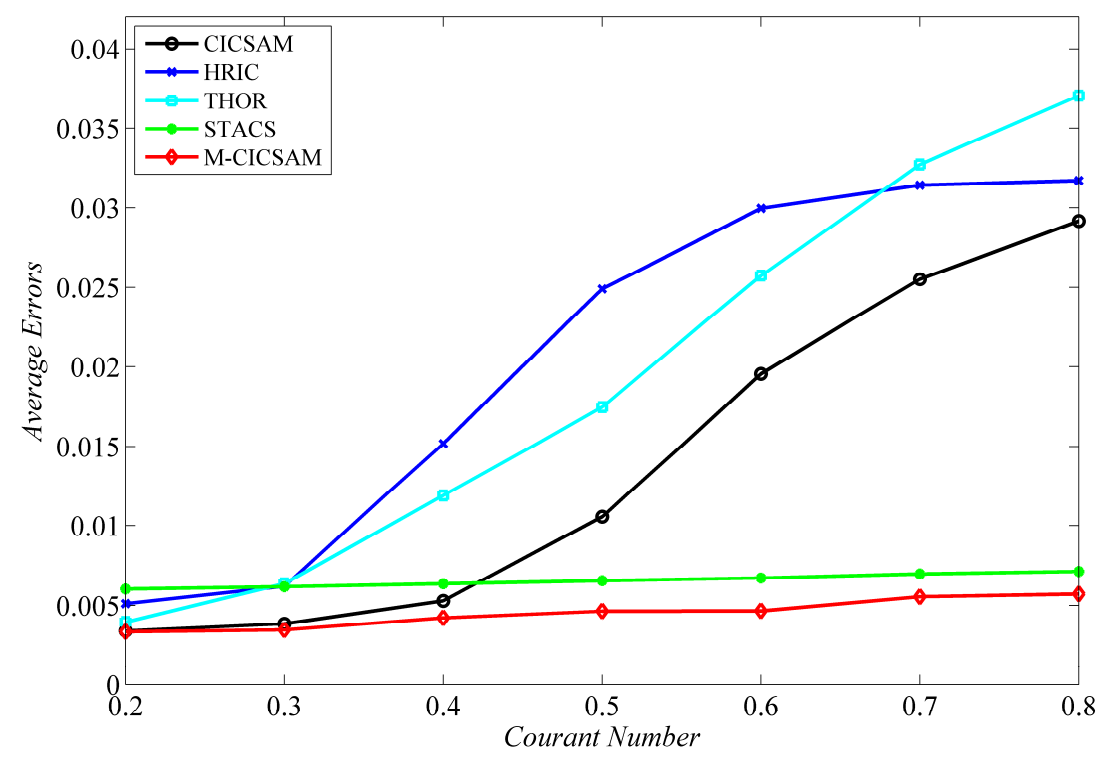

Figure 29. Comparison of accuracy of various blended high-resolution schemes at different Courant numbers in Test 2.

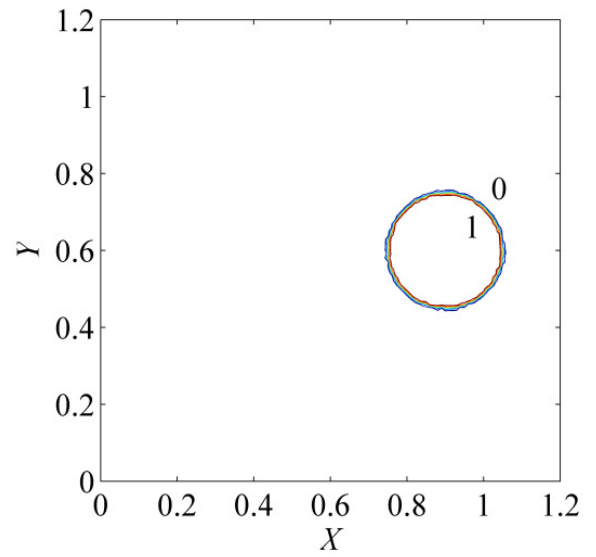

(a) The Exact Solution

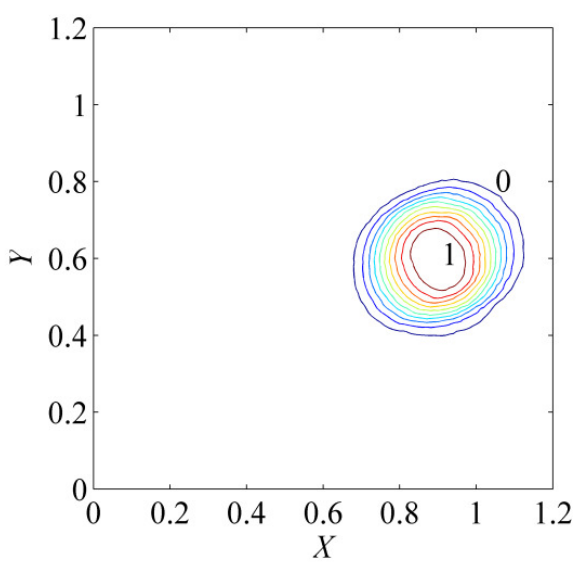

(b) HRIC

Figure 30. Contour plots of the advected round droplet at a Courant number of 0.6:

(a) the exact solution; (b) HRIC. 


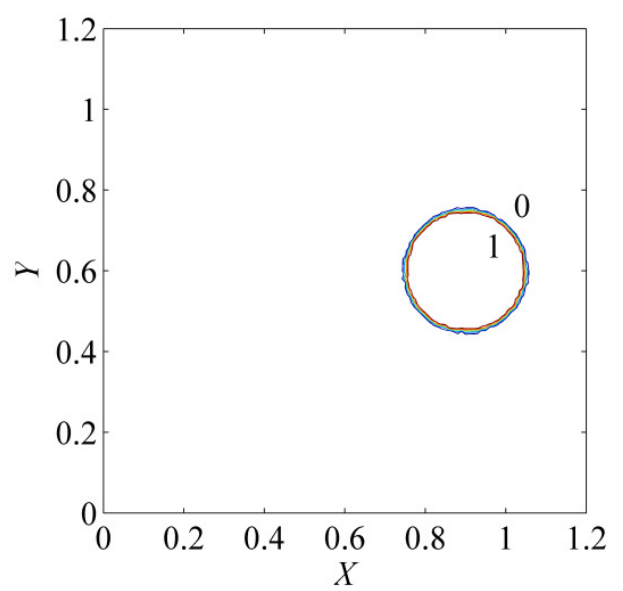

(a) The Exact Solution

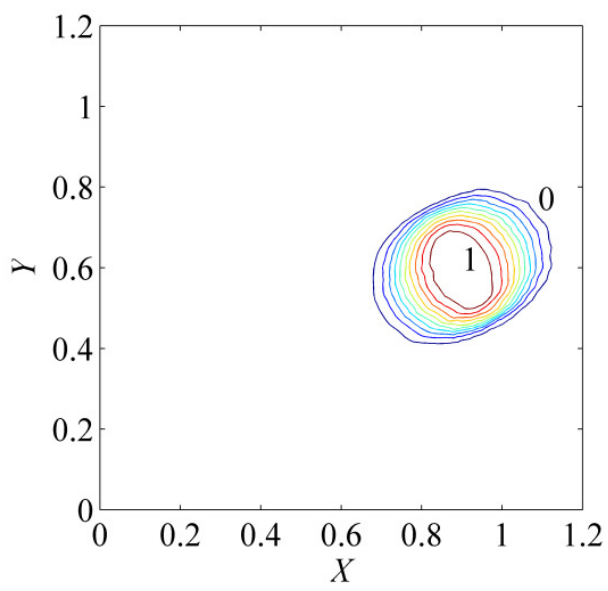

(b) THOR

Figure 31. Contour plots of the advected round droplet at a Courant number of 0.6: (a) the exact solution; (b) THOR.

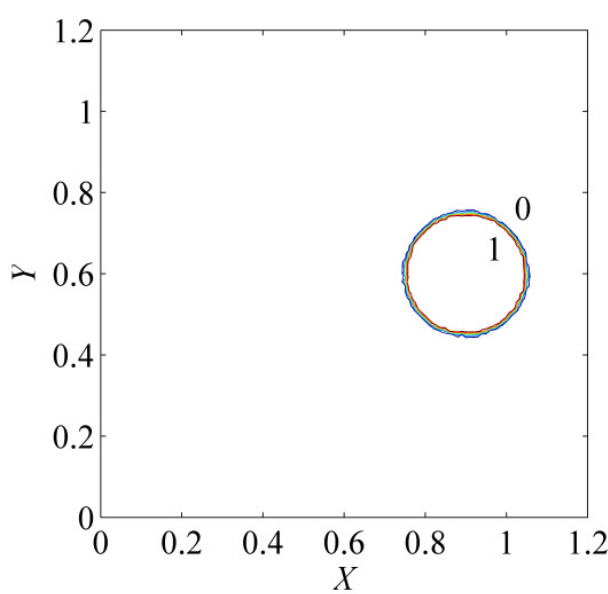

(a) The Exact Solution

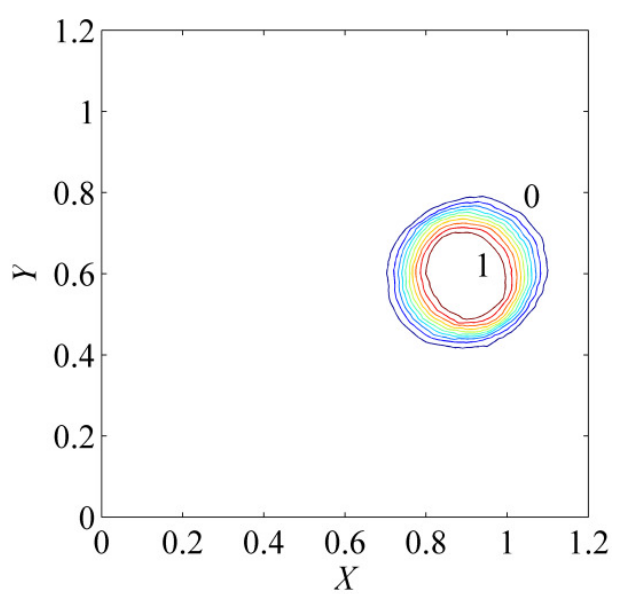

(b) CICSAM

Figure 32. Contour plots of the advected round droplet at a Courant number of 0.6: (a) the exact solution; (b) CICSAM.

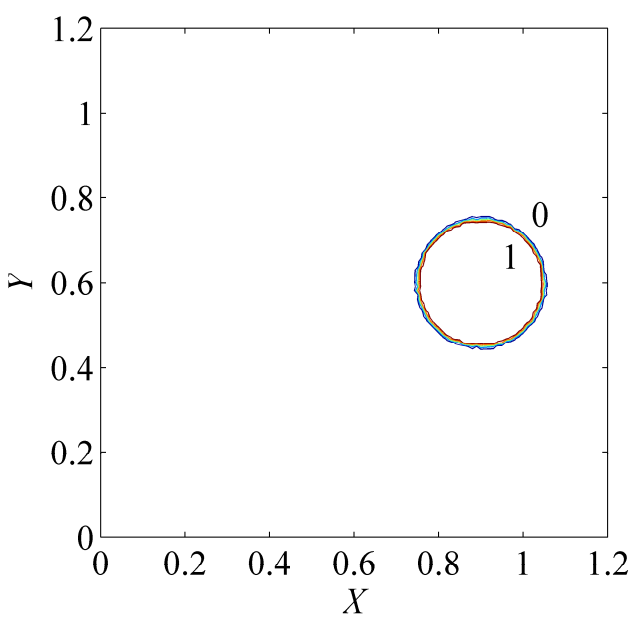

(a) The Exact Solution

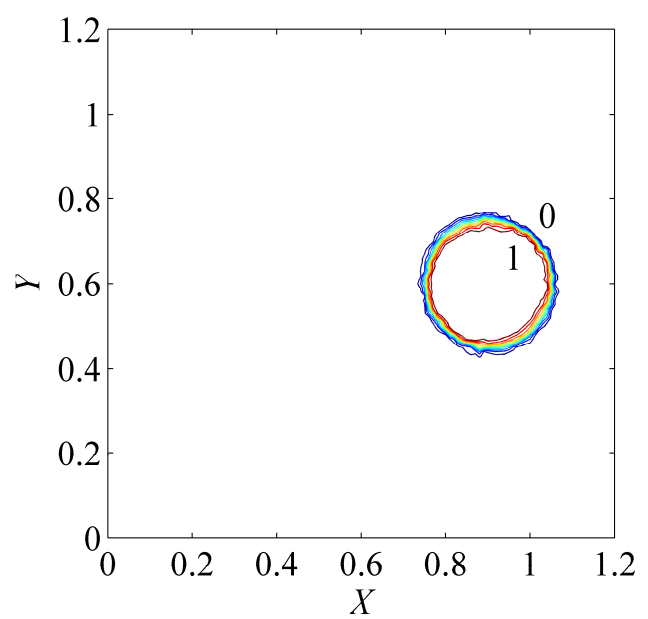

(b) STACS

Figure 33. Contour plots of the advected round droplet at a Courant number of 0.6: (a) the exact solution; (b) STACS. 


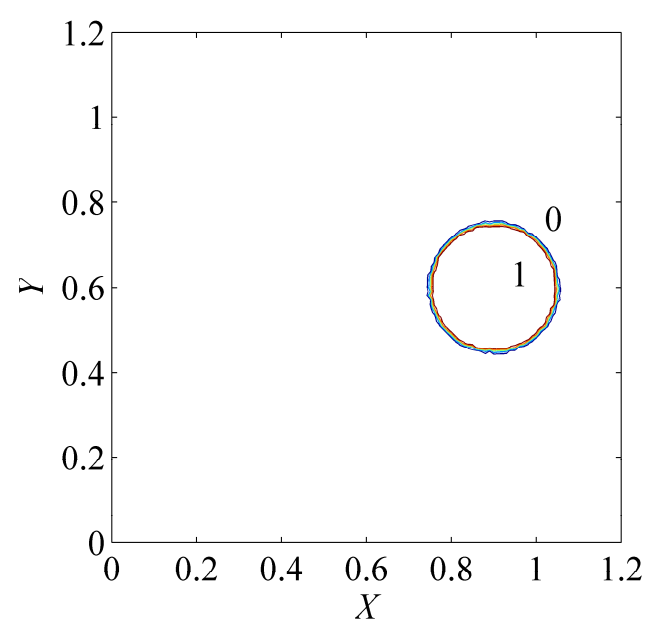

(a) The Exact Solution

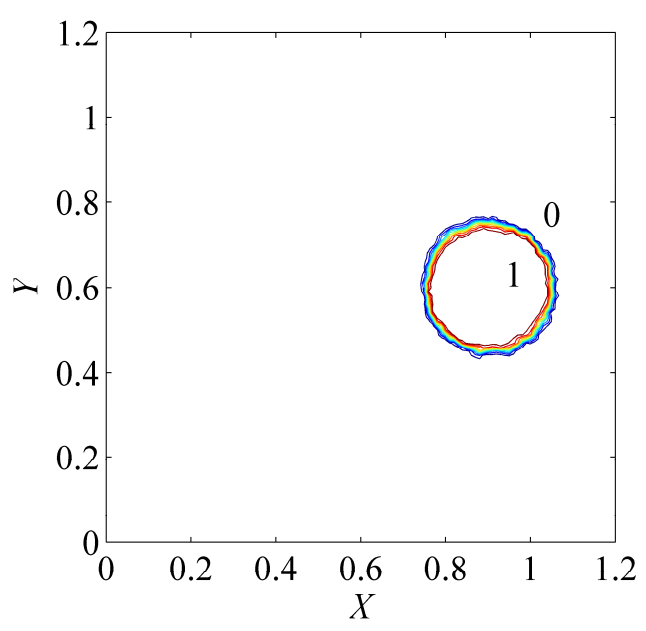

(b) M-CICSAM

Figure 34. Contour plots of the advected round droplet at a Courant number of 0.6:

(a) the exact solution; (b) M-CICSAM.

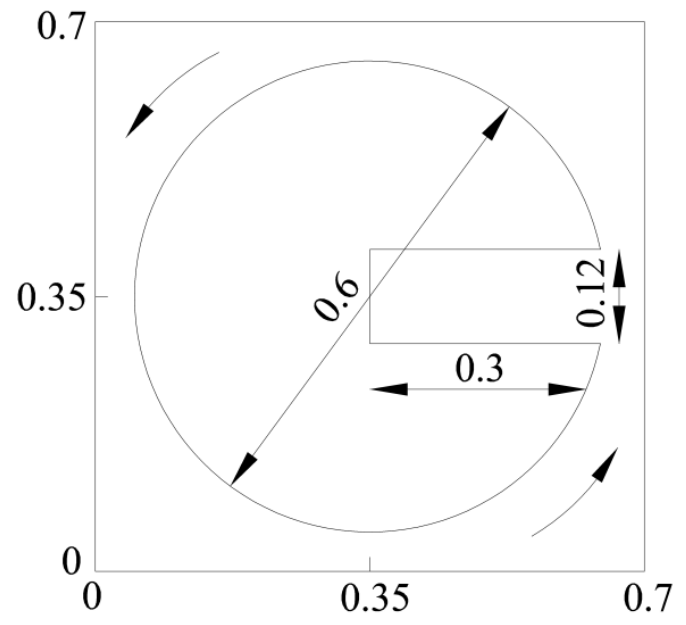

Figure 35. Schematic representation of the rotating slotted circle.

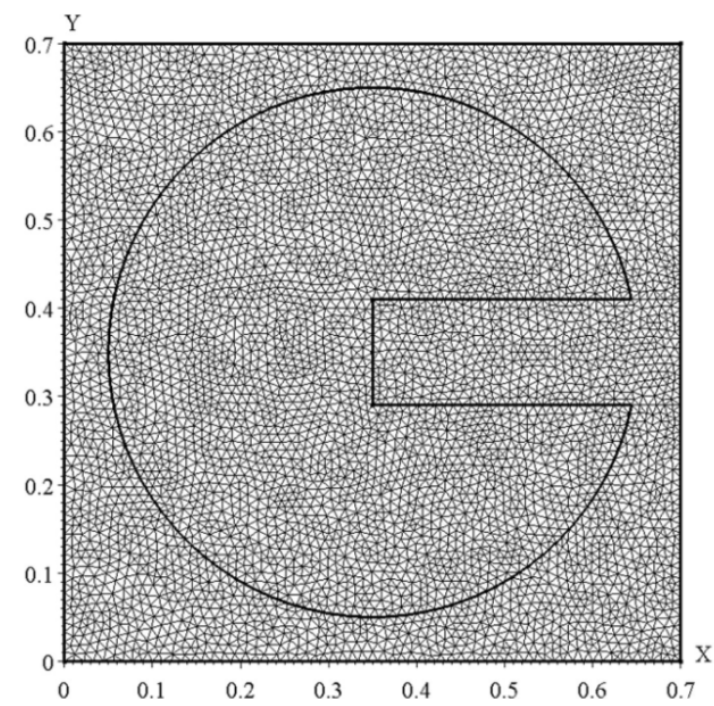

Figure 36. Physical domain and unstructured mesh used for the advection of a slotted circle in a rotational flow field. 


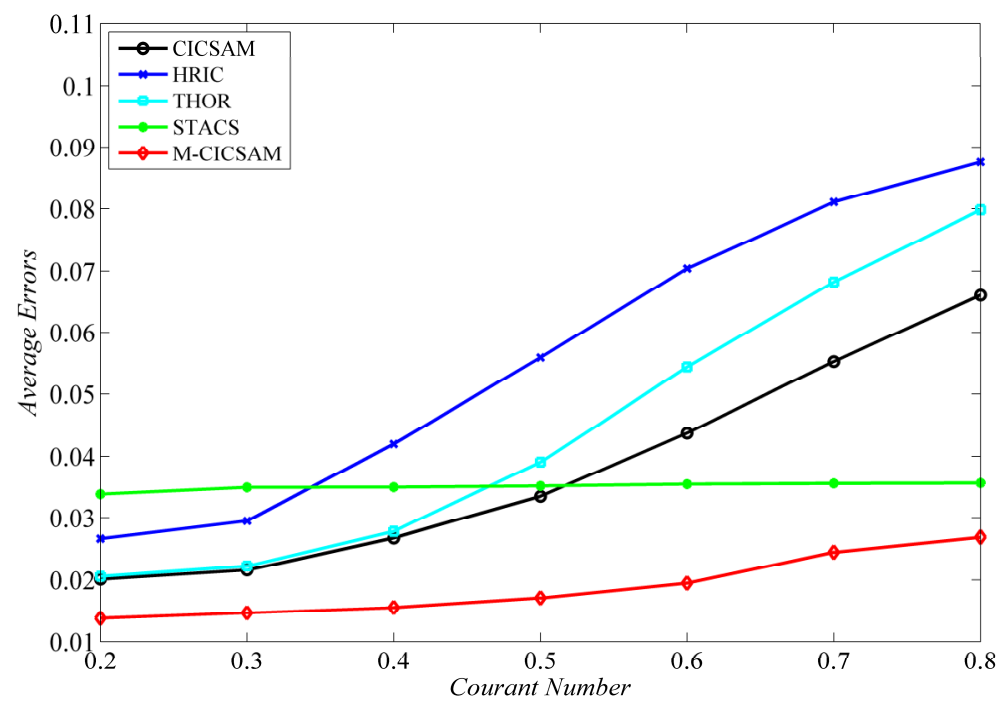

Figure 37. Comparison of accuracy of various blended high-resolution schemes at different Courant numbers in Test 3.

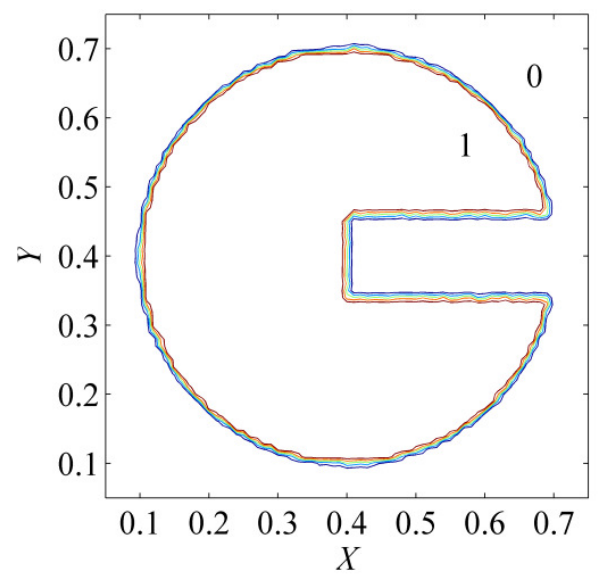

(a) The Exact Solution

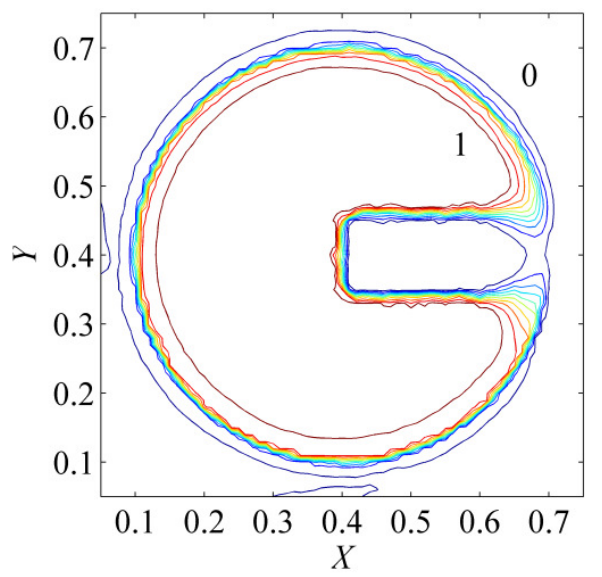

(b) HRIC

Figure 38. Contour plots of the rotating slotted circle at a Courant number of 0.5:

(a) the exact solution; (b) HRIC.

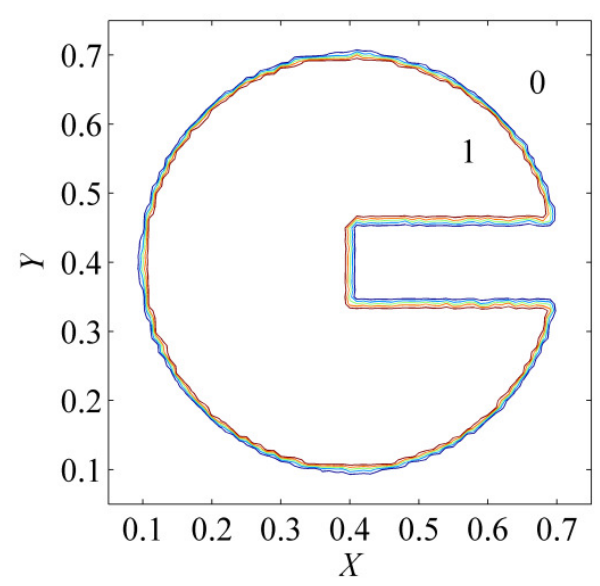

(a) The Exact Solution

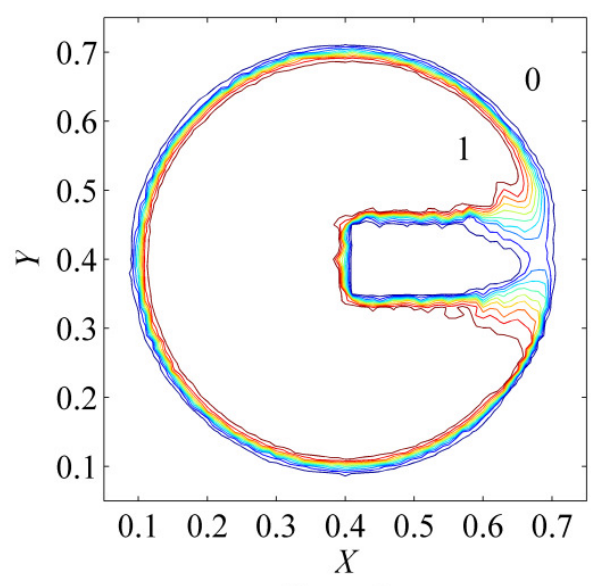

(b) THOR

Figure 39. Contour plots of the rotating slotted circle at a Courant number of 0.5: (a) the exact solution; (b) THOR. 


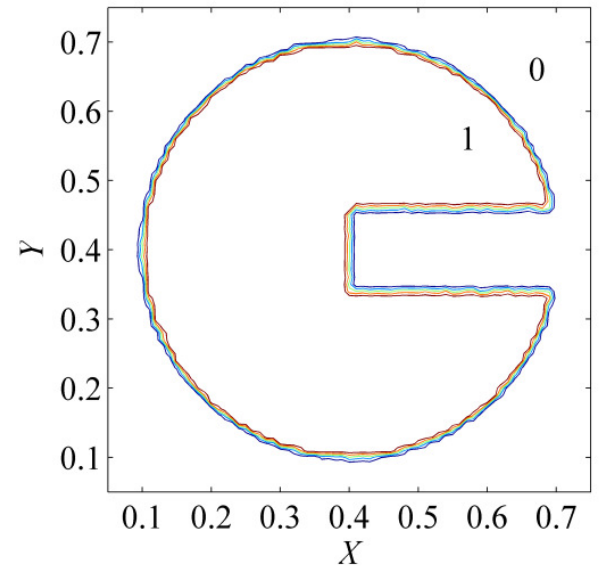

(a) The Exact Solution

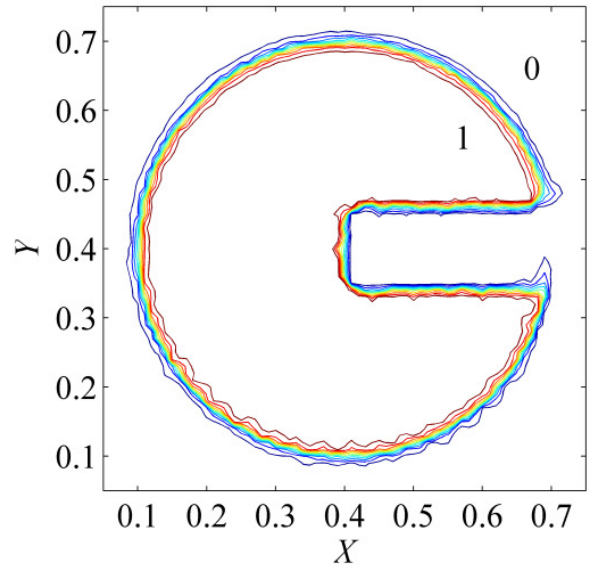

(b) CICSAM

Figure 40. Contour plots of the rotating slotted circle at a Courant number of 0.5:

(a) the exact solution; (b) CICSAM.

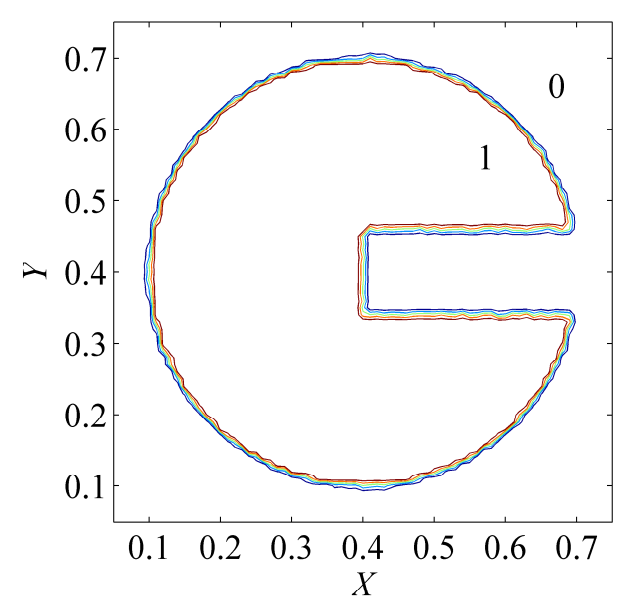

(a) The Exact Solution

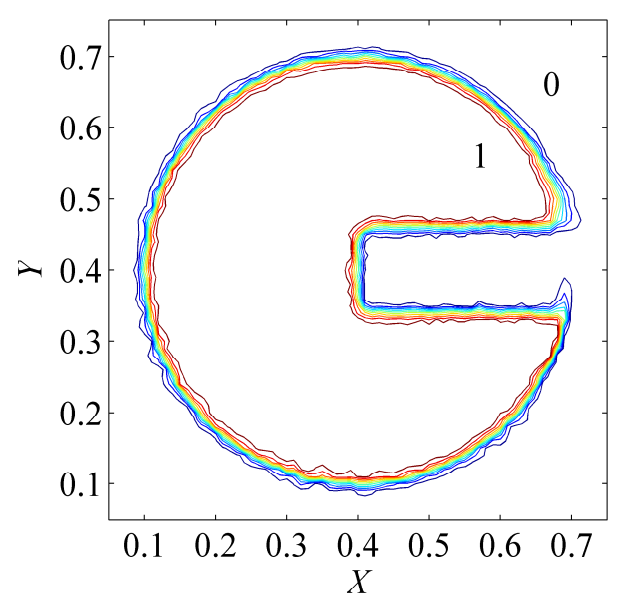

(b) STACS

Figure 41. Contour plots of the rotating slotted circle at a Courant number of 0.5: (a) the exact solution; (b) STACS.

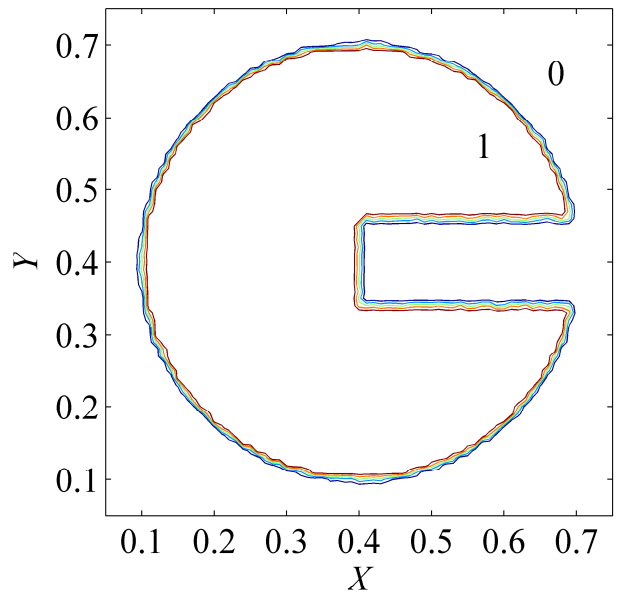

(a) The Exact Solution

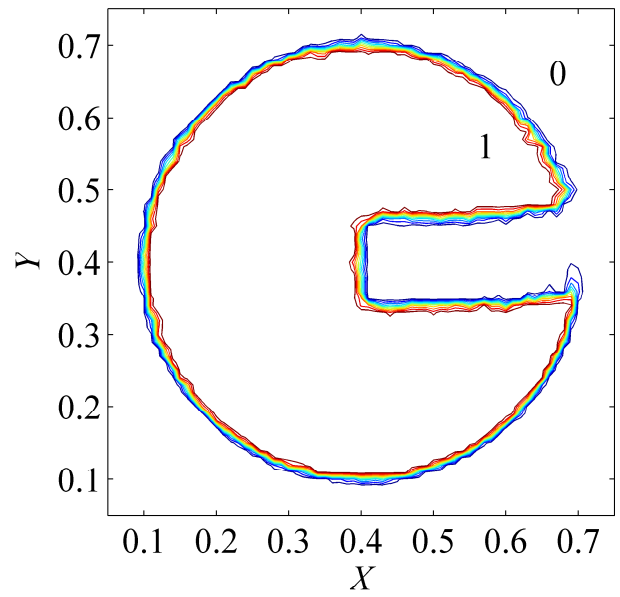

(b) M-CICSAM

Figure 42. Contour plots of the rotating slotted circle at a Courant number of 0.5: (a) the exact solution; (b) M-CICSAM. 


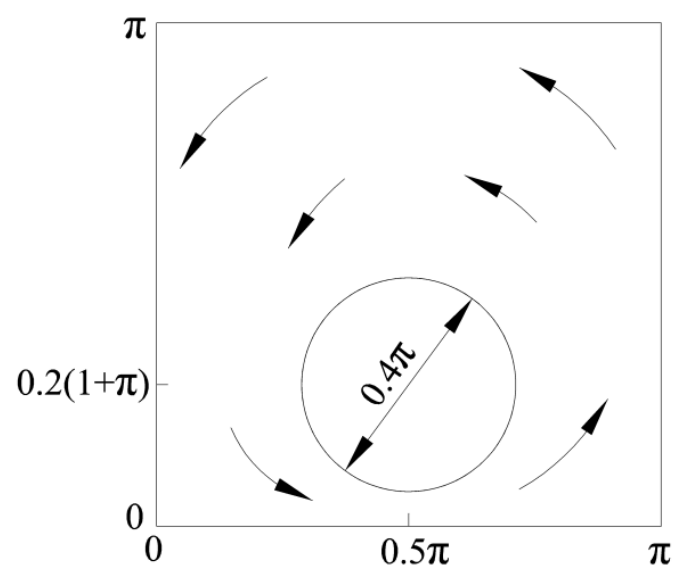

Figure 43. Schematic representation of the circle in shear flow field.

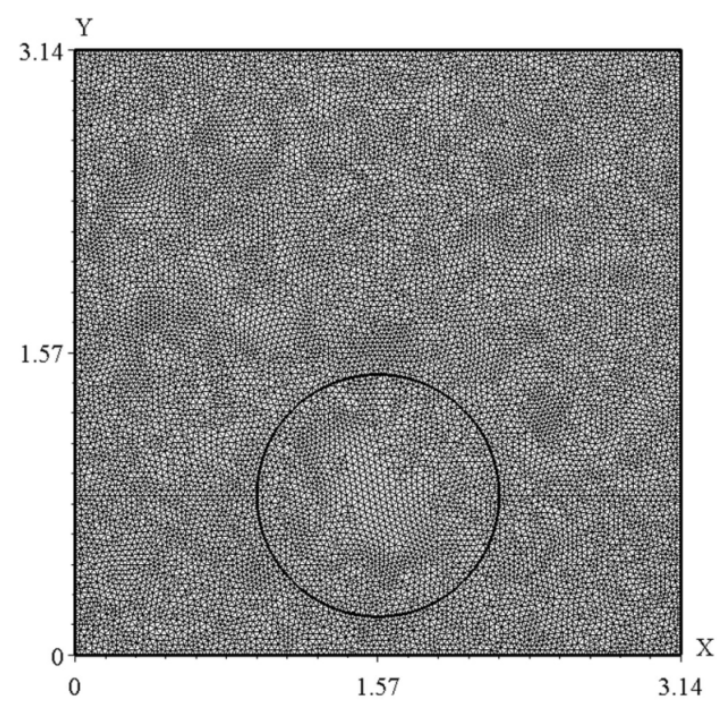

Figure 44. Physical domain and unstructured mesh used for the advection of a circle in a shear flow field.

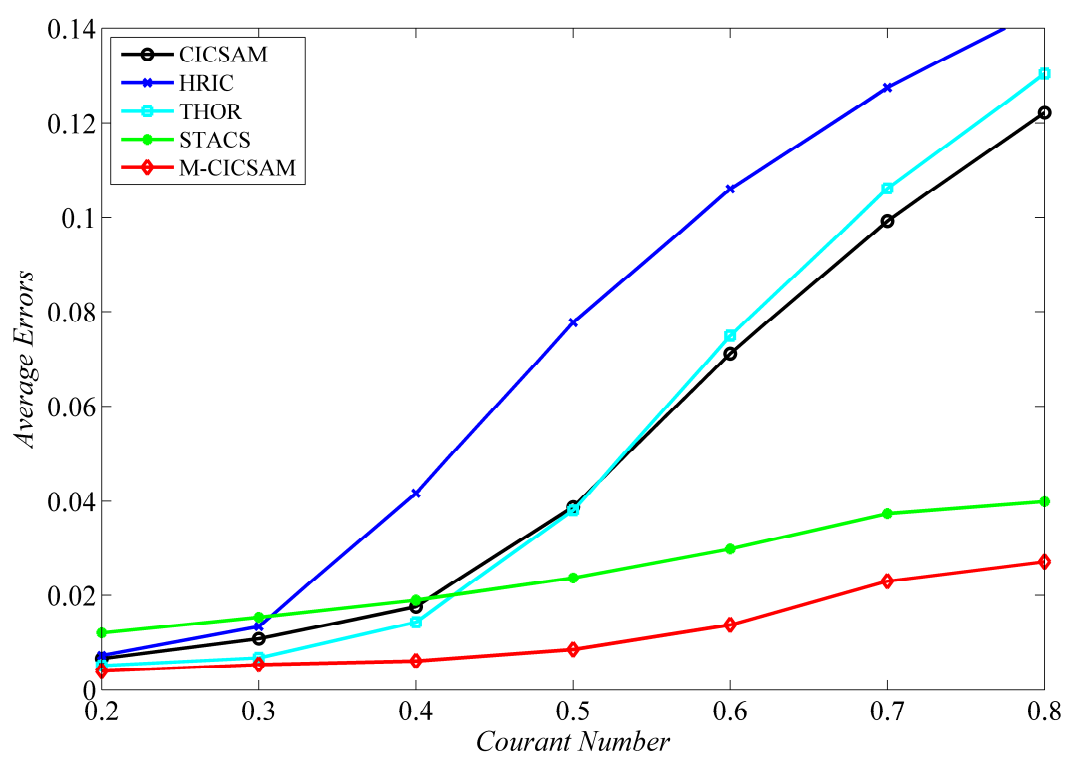

Figure 45. Comparison of accuracy of various blended high-resolution schemes at different Courant numbers in Test 4. 


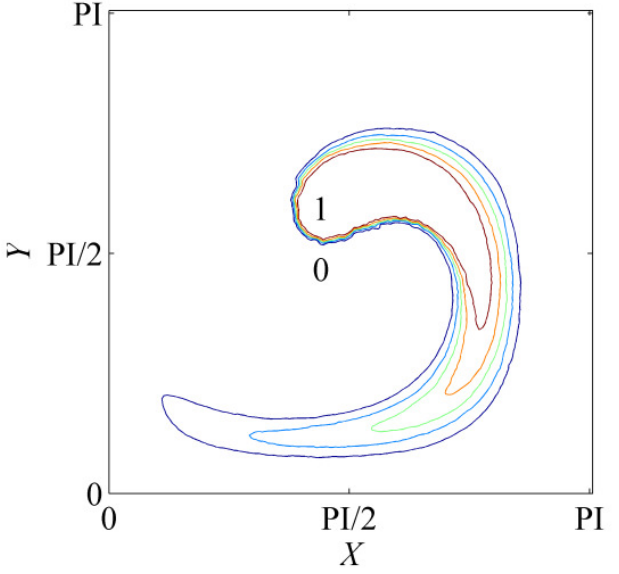

(a) HRIC - N=1000

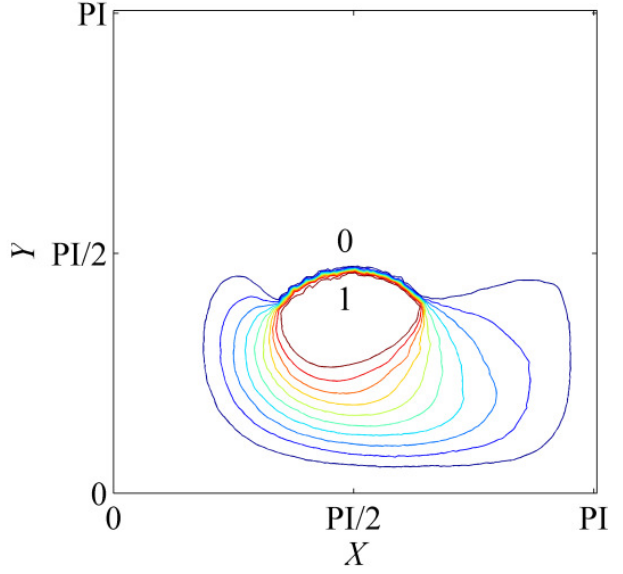

(b) HRIC - N=2000

Figure 46. Contour plots of the droplet in shear flow at a Courant number of 0.5 (HRIC): (a) after 1000 steps forward, (b) after 1000 steps forward followed by 1000 steps backward.

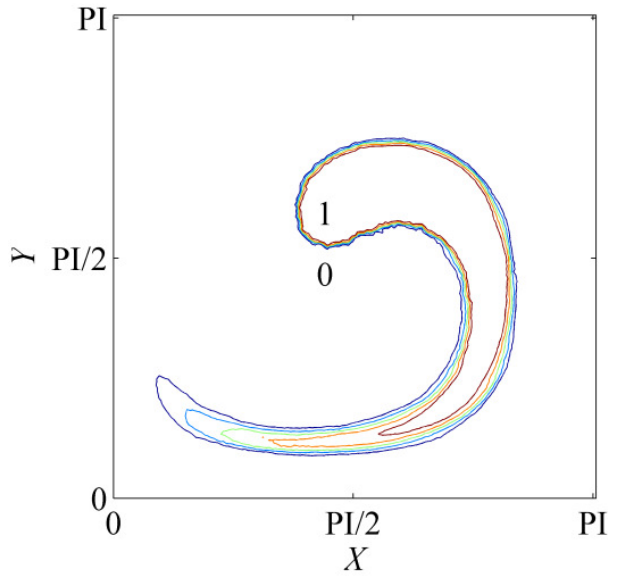

(a) THOR - N=1000

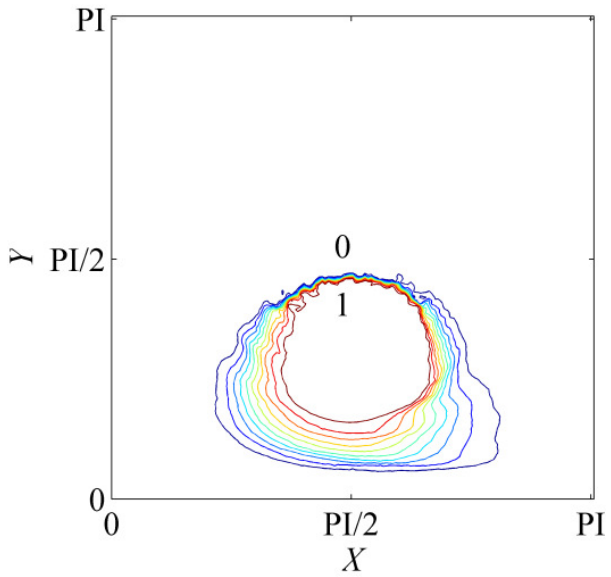

(b) THOR - N=2000

Figure 47. Contour plots of the droplet in shear flow at a Courant number of 0.5 (THOR): (a) after 1000 steps forward, (b) after 1000 steps forward followed by 1000 steps backward.

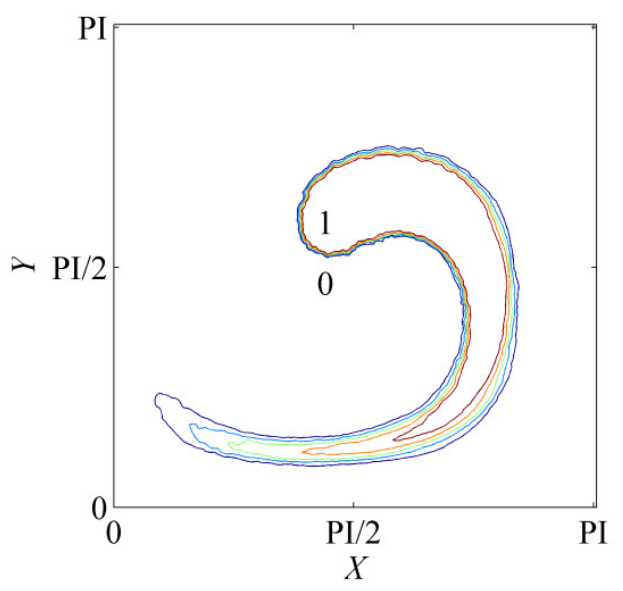

(a) CICSAM - N=1000

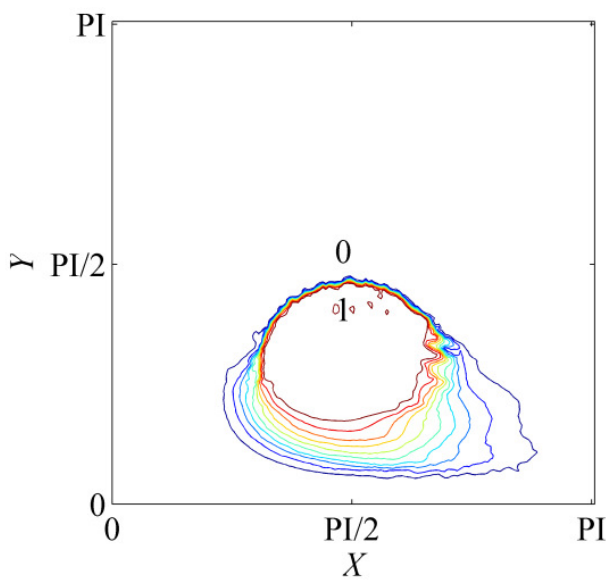

(b) CICSAM - N=2000

Figure 48. Contour plots of the droplet in shear flow at a Courant number of 0.5 (CICSAM): (a) after 1000 steps forward, (b) after 1000 steps forward followed by 1000 steps backward. 


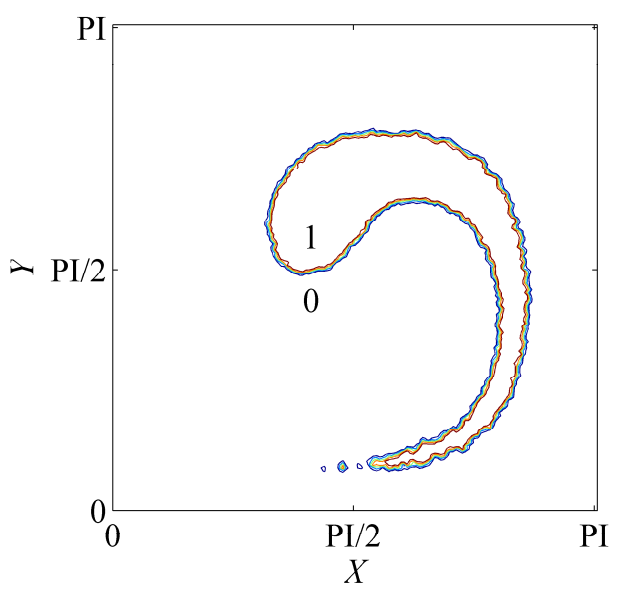

(a) STACS - N=1000

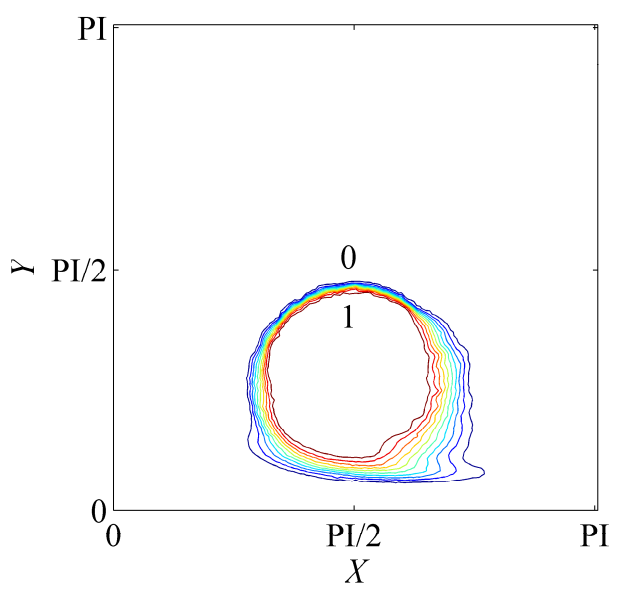

(b) STACS - N=2000

Figure 49. Contour plots of the droplet in shear flow at a Courant number of 0.5 (STACS): (a) after 1000 steps forward, (b) after 1000 steps forward followed by 1000 steps backward.

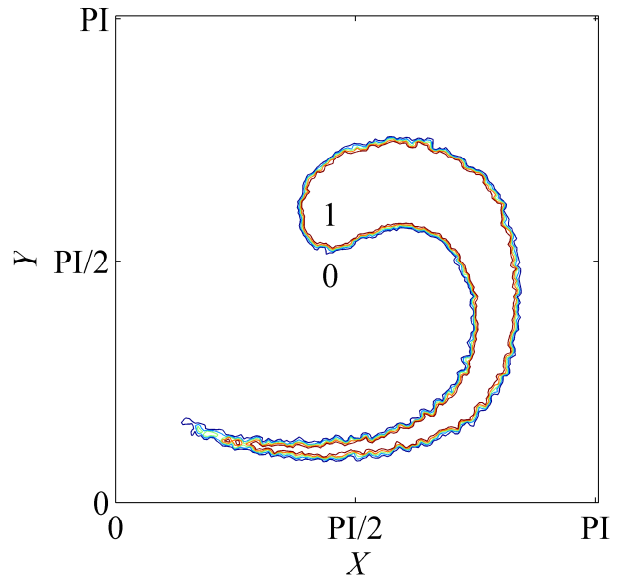

(a) M-CICSAM - N=1000

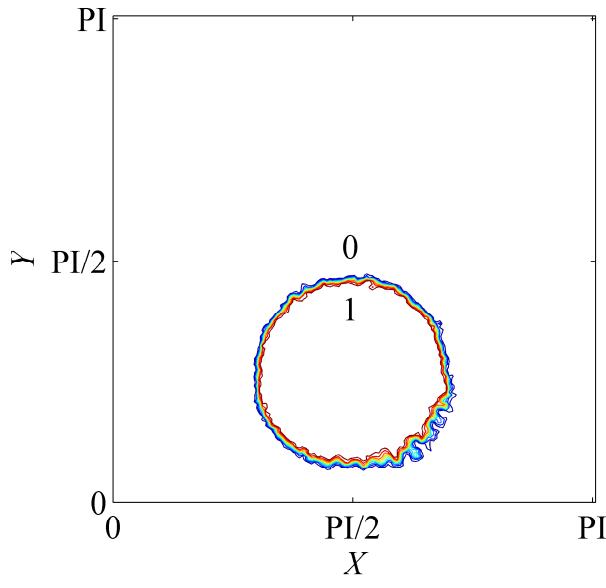

(b) M-CICSAM - N=2000

Figure 50. Contour plots of the circle in shear flow at a Courant number of 0.5 (M-CICSAM): (a) after 1000 steps forward, (b) after 1000 steps forward followed by 1000 steps backward. 